Boletín de la Sociedad Geológica Mexicana

VOLUMEN 62, NÚM 1, 2010, P. 173-185

\title{
Las cromititas del Complejo Ofiolítico de Camagüey, Cuba: un ejemplo de cromitas ricas en $\mathrm{Al}$
}

\author{
Saturnina Henares ${ }^{1, *}$, José María González-Jiménez ${ }^{2}$, Fernando Gervilla ${ }^{1}$, Joaquín A. Proenza ${ }^{3}$, \\ Alfonso Chang Rodríguez ${ }^{4}$, Rubén B. González-Pontón ${ }^{5 *}$
}

'Departamento de Mineralogía y Petrología e Instituto Andaluz de Ciencias de la Tierra (Universidad de Granada-CSIC), Facultad de Ciencias, Avda. Fentenueva s/n, 18002, Granada, España

${ }^{2}$ Key Centre Geochemical Evolution and Metallogeny of Continents (GEMOC), Department of Earth and Planetary Sciences, Macquarie University, Sydney NSW2109, Australia

${ }^{3}$ Departament de Cristal·lografia, Mineralogia i Dipòsits Minerals, Facultat de Geologia, Universitat de Barcelona, Martí i Franquès s/n, 08028, Barcelona, España

${ }^{4}$ Cuban Geological Survey. Ave. Mónaco Este e/ 1ra y B, Edif. 38 Apto 4, Rpto. Julio A. Mella, Camagüey, Cuba

${ }^{5}$ Empresa Geominera de Camagüey, Carretera Central Este, km 51/2 70600, Camagüey, Cuba

* Corresponding author: E-mail: jmgonzj@ugr.es

Resumen

El Complejo Ofiolítico de Camagüey se sitúa en la región centro-oriental de la Isla de Cuba. Este complejo ofiolítico contiene importantes depósitos de cromita rica en $\mathrm{Al}[\mathrm{Cr} /(\mathrm{Cr}+\mathrm{Al})=0.31-0.6]$ y $\mathrm{TiO}_{2}$ (hasta $1 \%$ en peso), y representa el segundo distrito minero en importancia de cromita de grado refractario de Cuba. Los cuerpos de cromititas se encuentran encajados en dunitas y harzburgitas muy próximas a los niveles de cumulados gabroicos de la base de la corteza plutónica, donde se encuentran espacialmente asociados con rocas feldespáticas (diques y/o sills de gabros, troctolitas y/o anortositas). La textura predominante en los depósitos de cromita es la masiva, aunque también se reconocen diseminada, nodular y brechoide. La matriz intersticial de la cromitita está constituida, mayoritariamente, por olivino y en menor medida por piroxenos y plagioclasa. A menudo estos minerales se encuentran alterados a otros minerales secundarios tales como serpentina, clorita, magnetita y granate tipo uvarovita. Los cristales de cromita contienen pequeñas inclusiones sólidas de los silicatos que forman la matriz, óxidos de Fe, Ti y/o Zr y sulfuros y aleaciones de Fe-Ni-Cu. Estos últimos minerales también se encuentran en la matriz alterada, mostrando evidencias de alteración. Las características estructurales, petrológicas y geoquímicas de las cromititas del Complejo Ofiolítico de Camagüey sugieren que podrían tener su origen a partir de fundidos generados tras la reacción entre fundidos basálticos y peridotitas en el manto superior litosférico, o bien como producto de la asimilación, por parte de los fundidos basálticos, de rocas feldespáticas (gabros y troctolitas) localizadas en las zonas más someras del manto superior.

Palabras claves: Cromitita rica en Al, Complejo Ofiolítico, Camagüey, Cuba.

Abstract

The Camagüey Ophiolitic Complex extends along the central-eastern region of Cuba. This ophiolitic complex contains several deposits of Al-rich chromite $[\mathrm{Cr} /(\mathrm{Cr}+\mathrm{Al})=0.31-0.6]$ very enriched in $\mathrm{TiO}_{2}$ (up to $1 \mathrm{wt} \%$ ) and represents the second most important mining district for refractory grade chromite in Cuba. The chromite deposits are hosted by dunite and harzburgite, spatially associated with feldspatic rocks (gabbro dikes and/or sills, troctolites and/or anorthosites) in close proximity to gabbroic cumulates at the base of the plutonic crust. The texture observed in the chromite deposits is predominantly massive, although fine disseminated, nodular and brecciated textures are also present. The interstitial silicate matrix of the chromitite consists principally of olivine and to a lesser extent pyroxenes and plagioclase. Often these silicates are altered to other secondary minerals such as serpentine, chlorite, magnetite and uvarovite-type garnet. The chromite crystals usually host small solid inclusions of the same silicates that form the matrix, Fe-, Ti-and/or Zr-rich oxides, and Fe-Ni-Cu sulphides and alloys. The above mentioned metallic minerals also occur in the interstitial silicate matrix where they record variable alteration. The structural, petrological and geochemical characteristics of the chromitites suggest formation from melts generated after reaction of basaltic melts with upper lithospheric mantle peridotites or, alternatively, as result of the assimilation of pre-existing feldspatic rocks (gabbros and troctolites) located in the shallowest upper mantle.

Keywords: Al-rich Chromitite, Ophiolitic Complex, Camagüey, Cuba. 


\section{Introducción}

La cromita que forma la mayoría de los cuerpos de cromititas ofiolíticas ("cromititas podiformes") es rica en $\mathrm{Cr}[\# \mathrm{Cr}=\mathrm{Cr} /(\mathrm{Cr}+\mathrm{Al}) \geq 0.6]$. No obstante, también existen ejemplos de complejos ofiolíticos en los cuales se encuentran cuerpos podiformes de cromititas ricas en $\mathrm{Al}(\# \mathrm{Cr} \leq 0.6)$. Las cromititas ricas en $\mathrm{Cr}$ encajan, generalmente, en dunitas representativas de porciones relativamente profundas del manto superior (Leblanc y Violette, 1983; Bédard y Hébert, 1998; Proenza et al., 1999a, 1999b; Ahmed y Arai 2003; Gervilla et al., 2005). En cambio, las cromititas ricas en Al mayoritariamente suelen estar restringidas a la zona de transición manto-corteza, o Moho Transition Zone (MTZ), próximas a los niveles de gabros bandeados de la base de la corteza plutónica (Leblanc y Violette, 1983; Proenza et al., 1999a, 1999b).

Los depósitos ofiolíticos de cromita representan una parte muy importante de la producción mundial de cromo. Además, estos depósitos constituyen la única fuente natural de cromita de grado refractario o rica en $\mathrm{Al}$ (cromita con $\mathrm{Al}_{2} \mathrm{O}_{3}>20 \%$ en peso, $\left(\mathrm{Al}_{2} \mathrm{O}_{3}+\mathrm{Cr}_{2} \mathrm{O}_{3}\right)>60 \%$ en peso y con bajo contenido de hierro).

El Complejo Ofiolítico de Camagüey (Cuba) contiene importantes reservas de cromita rica en $\mathrm{Al}$ y constituye el segundo distrito más importante de cromita de grado refractario de Cuba (Thayer, 1942; Flint et al., 1948; Kravchenco y Vázquez, 1985; Lavandero et al., 1988; Murashko y Lavandero, 1989; Rodríguez et al., 1997; Proenza y Melgarejo, 1998; González-Pontón, 1998, 2009). En este distrito se han descrito más de 340 yacimientos y manifestaciones de cromita, siendo el yacimiento Camagüey-II el más grande, con reservas que exceden las 700,000 t (González-Pontón, 1998). Otros depósitos tienen reservas superiores a 100,000 $\mathrm{t}$ (e. g. Victoria I) o a 50,000 t (e.g. Mamina, Lolita, Ofelia Ferrolana; González-Pontón, 2009).

Los cuerpos de cromitita de Camagüey encajan en una MTZ dominada por la presencia de rocas ultramáficas (harzburgitas, lherzolitas y dunitas), junto con numerosos cuerpos de rocas feldespáticas (gabros, gabros olivínicos, noritas, troctolitas y anortositas). La mayoría de los cuerpos de cromitita presentan una envolvente dunítica de espesor variable y, a menudo, se encuentran cortados por diques de gabro y/o troctolitas (Thayer, 1942; Flint et al., 1948). No está claro, sin embargo, que las cromititas contengan cuerpos de gabros concordantes (sills), análogos a los descritos por Proenza et al. (1999a, 1999b) en el Complejo de Moa-Baracoa, Cuba oriental.

Dada la importancia metalogenética del distrito minero de Camagüey y la escasez de estudios sistemáticos de los depósitos de cromita, en este trabajo se pretende: i) analizar las características morfológicas de los cuerpos de cromititas y sus relaciones con las rocas encajantes, ii) caracterizar los tipos texturales de cromitita, desde un punto de vista mineralógico y de su química mineral, iii) estudiar las variaciones composicionales de la cromita en función de su asociación a rocas máficas (gabros) o ultramáficas (dunitas), y finalmente, iv) establecer, en la medida de lo posible, un modelo conceptual de formación para las cromititas.

\section{Contexto Geológico}

La Geología del Norte de la Isla de Cuba está caracterizada por la presencia de varios terrenos ofiolíticos que constituyen el denominado Cinturón Ofiolítico Cubano Septentrional (Figura 1A; Iturralde-Vinent, 1989, 1996, 1998; GarcíaCasco et al., 2006, 2008). Este cinturón ofiolítico, del Jurásico Superior-Cretácico Inferior, se puede dividir en tres terrenos principales (Khudoley y Meyerhoff, 1971; Knipper y Cabrera, 1974; Albear e Iturralde-Vinent, 1985): 1) Cinturón Ofiolítico de Cajálbana, al Oeste, 2) Cinturón Ofiolítico de Mayarí-Baracoa, al Este y 3) Cinturón Ofiolítico de MarielHolguín, en la parte central de la Isla de Cuba (Figura 1A). En este Cinturón Ofiolítico Septentrional se han descrito todos los niveles de una secuencia ofiolítica ideal, aunque muy desmembrada tectónicamente (Iturralde-Vinent, 1996). El Cinturón Ofiolítico de Mariel-Holguín está compuesto por los macizos ofiolíticos de Holguín, Camagüey, Matanzas, La Habana y Mariel. La unidad mantélica está dominada por harzburgitas que albergan cuerpos de dunitas y lherzolitas, sobre la cual se dispone una unidad de gabros bandeados e isotrópicos, subyacente a un complejo volcano-sedimentario que aflora raramente (Fonseca et al., 1985; Iturralde-Vinent, 1989; Andó et al., 1996).

El Complejo Ofiolítico de Camagüey está situado en la región centro-oriental del Cinturón Ofiolítico de MarielHolguín (Figura 1A) y presenta una morfología en forma de arco de, aproximadamente, $120 \mathrm{~km}$ de longitud y anchura máxima de $40 \mathrm{~km}$ (promedio $10 \mathrm{~km}$ ). El Complejo Ofiolítico de Camagüey se extiende a lo largo de $1200 \mathrm{~km}^{2}$. Está formado por dos estructuras antiformes, en las cuales se reconoce una secuencia ofiolítica casi completa. Toda la estructura del complejo ofiolítico cabalga sobre los sedimentos mesozoicos de la Plataforma Norteamericana (Figura 1B; IturraldeVinent, 2001). La unidad de peridotitas está constituida, principalmente, por harzburgitas con cantidades menores de websteritas, lherzolitas y cuerpos dispersos de dunitas (Figura 2). Las harzburgitas muestran un tamaño de grano medio y están compuestas por olivino y ortopiroxeno (enstatita, 8-10\% en volumen) alterados, en su mayoría, a serpentina. Las dunitas pueden contener pequeñas cantidades de plagioclasa, sin alcanzar nunca el $10 \%$ en volumen, y espinela cromífera accesoria (Flint et al., 1948). Hacia la parte alta de esta unidad mantélica se reconocen abundantes cuerpos de rocas feldespáticas en forma de masas irregulares de gran tamaño, diques y/o cuerpos concordantes. Las rocas feldespáticas más abundantes son las troctolitas y, en menor medida, los gabros (en ocasiones ricos en olivino, con tamaño de grano que varía desde muy fino a gabro pegmatitas) y las anortositas. Por último, la unidad volcano-sedimentaria está constituida por basaltos de edad albiense?-cenomaniense?, hialoclastitas, pedernales radiolaríticos y limonitas (Figura 2; IturraldeVinent, 1996, 2001).

\section{Técnicas Analíticas}

Se han estudiado un total de 20 muestras de 6 cuerpos de cromititas mediante microscopía de luz reflejada y transmitida, 


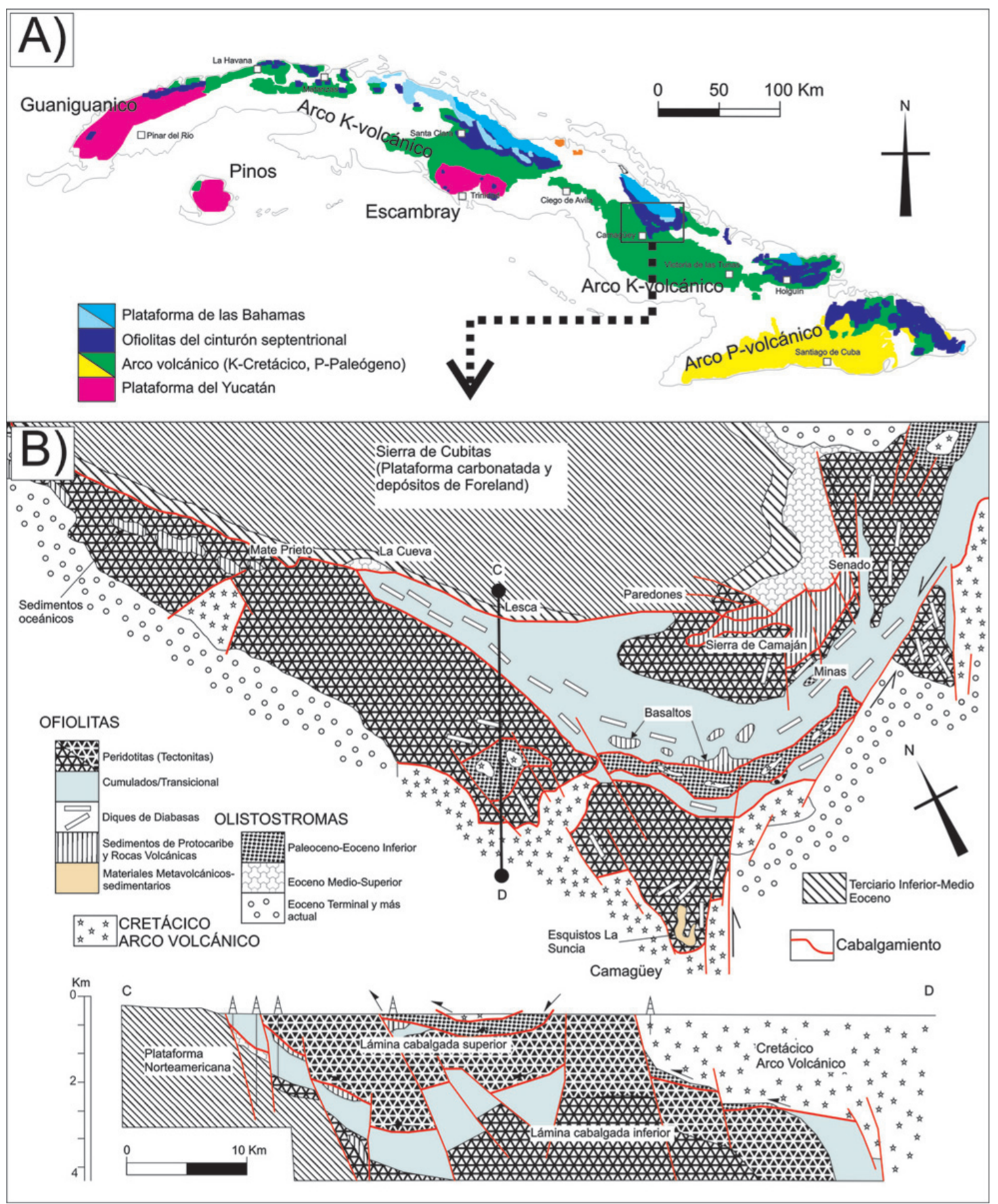

Figura 1. A) Localización de las ofiolitas del Cinturón Ofiolítico Cubano Septentrional. B) Estructura de las ofiolitas de Camagüey, Cuba (modificado de IturraldeVinent, 2001).

microscopía electrónica de barrido con analizador EDX asociado y microsonda electrónica.

El estudio mediante microscopía electrónica de barrido se llevó a cabo en modo ambiental (ESEM) utilizando un equipo modelo Philips Quanta 400 con un detector EDX acoplado a un ordenador con software EDAX, perteneciente al Centro Andaluz de Medio Ambiente, Junta de Andalucía-Universidad de Granada. Las inclusiones sólidas presentes en los granos de cromita fueron analizadas de forma semicuantitativa usando espectrómetros de dispersión de energía (EDS) y un tiempo de conteo medio de 35 segundos. El objeto de este estudio fue la caracterización química de estas inclusiones, así como la obtención de microfotografías de electrones retrodispersados y de electrones secundarios de granos minerales de tamaños inferiores, incluso, a 1 micrómetro $(1 \mu \mathrm{m})$.

El análisis cuantitativo de la cromita y de los silicatos se llevó a cabo con dos microsondas CAMECA SX50 y SX100 pertenecientes, respectivamente, a los Serveis Cientificotécnics de la Universitat de Barcelona y al Centro de Instrumentación Científica de la Universidad de Granada. En ambos casos, las 
condiciones de trabajo utilizadas fueron: voltaje de aceleración de $20 \mathrm{kV}$, corriente de sonda sobre la muestra de 15-20 nA y diámetro del haz incidente de $2 \mu \mathrm{m}$. Los patrones de calibración utilizados en el primer caso fueron ortoclasa $(\mathrm{Si}, \mathrm{Al}, \mathrm{K}), \mathrm{Fe}_{2} \mathrm{O}_{3}$ $(\mathrm{Fe})$, periclasa $(\mathrm{Mg})$, wollastonita $(\mathrm{Ca})$, rodonita $(\mathrm{Mn}), \mathrm{NiO}$ $(\mathrm{Ni})$, rutilo (Ti), albita $(\mathrm{Na}), \mathrm{Cr}_{2} \mathrm{O}_{3}(\mathrm{Cr})$ y esfalerita $(\mathrm{Zn})$. En el segundo caso, los patrones utilizados fueron los mismos excepto sílice pura para el $\mathrm{Si}, \mathrm{Al}$-espinela para el $\mathrm{Al}$, sanidina para el $\mathrm{K}$ y vanadinita para el V. La fórmula estructural de la cromita fue calculada por estequiometría siguiendo el procedimiento descrito por Carmichael (1967).

\section{Características de las cromititas del Complejo Ofiolítico de Camagüey}

4.1. Morfología de los cuerpos de cromitita y relaciones con las rocas encajantes

En el Complejo Ofiolítico de Camagüey las cromititas encajan en peridotitas que contienen abundantes cuerpos de rocas feldespáticas (Figura 2). El tamaño de los cuerpos de cromitita varía desde pequeñas concentraciones de forma nodular hasta grandes cuerpos que llegan a superar las 200,000 t (Flint et al., 1948). En su mayoría, presentan forma lenticular o de pod, aunque también se reconocen cuerpos equidimensionales, irregulares y tabulares. Los cuerpos de cromitita encajados en harzburgitas muestran una envolvente dunítica que varía desde unos pocos centímetros a varios metros. El contacto entre la cromitita y el encajante (en su mayoría dunita) es, generalmente, gradual (Thayer, 1942; Flint et al., 1948) aunque algunos de los cuerpos de cromitita están delimitados o afectados por fallas.

Los cuerpos de cromitita son, por lo general, subhorizontales (Flint et al., 1948; Semionov, 1968); sin embargo, también se han descrito cuerpos subverticales, como en el caso de las Minas Victoria y Camagüey (Flint et al., 1948; Kravchenco y Vázquez, 1985; Murashko y Lavandero, 1989). Los cuerpos lenticulares y tabulares tienden a ser paralelos a los contactos entre las peridotitas serpentinizadas y los gabros (Thayer, 1942; Flint et al., 1948; Semionov, 1968; Kravchenco y Vázquez, 1985; Murashko y Lavandero, 1989). Es frecuente observar la presencia de diques de diversa naturaleza que cortan a los cuerpos de cromitita (Figura 3). Los diques de gabros pegmatíticos son poco frecuentes dentro y cerca de los pods de cromititas, observándose en algunas localidades (e. g. Mina Aventura). Los diques de gabro de tamaño de grano más fino se encuentran, comúnmente, en las harzburgitas (Flint et al., 1948). Las troctolitas se presentan como grandes cuerpos, bien en forma de capas inclinadas, o como diques tabulares y verticales, que cortan a las cromititas y a las peridotitas. Dentro de estos diques se reconocen, con frecuencia, fragmentos sueltos y brechas cementadas de cromitita (Flint et al., 1948). A veces se encuentran segregaciones de poca potencia de dunitas en los cuerpos de cromititas (Figura 3).

\subsection{Texturas de las cromititas}

Las cromititas del Complejo Ofiolítico de Camagüey presentan gran variedad de texturas: masiva, diseminada,

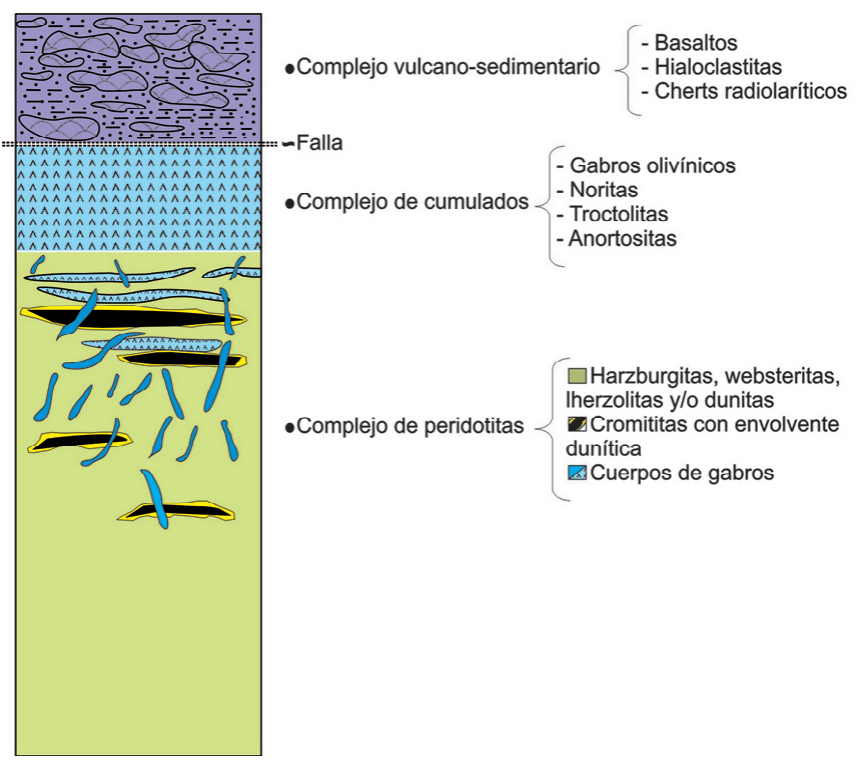

Figura 2. Representación esquemática de la columna litológica del Complejo Ofiolítico de Camagüey, Cuba (modificado de Iturralde-Vinent, 1989).

nodular y brechoide. La textura masiva ( $>90 \%$ en volumen de cromita) es la que constituye la mayor parte de los cuerpos minerales. De manera similar a lo que ocurre en otras cromititas ofiolíticas (Thayer, 1942; Leblanc y Nicolas, 1992), a medida que aumenta el contenido en silicatos (hacia las zonas exteriores de los cuerpos en contacto con el encajante) la textura pasa a ser diseminada. Dicha textura es la más frecuente en los bordes de los cuerpos y es la que predomina, también, en los cuerpos de cromitita de menor tamaño y/o morfología irregular. En las texturas nodulares, muchos de estos nódulos presentan cierto grado de deformación mecánica. Por lo general, presentan un tamaño de grano que no supera los $2 \mathrm{~cm}$ de diámetro. En el caso de la Mina Guillermina, los nódulos de cromita están embebidos en una matriz troctolítica en la zona central del depósito y dunítica hacia los bordes. Dentro de estos nódulos se identifican cristales de plagioclasa parcialmente alterada (Figura 4). La textura brechoide es común y consiste en fragmentos de rocas, de tamaño superior a $2.5 \mathrm{~cm}$, de morfología variada: desde clastos angulosos hasta piezas redondeadas. Como regla general, los fragmentos son equidimensionales y no están orientados. Sin embargo, en la Mina Aventura los fragmentos de cromita se presentan elongados según una orientación preferente.

\subsection{Mineralogía de las cromititas}

Los principales minerales que forman las cromititas del Complejo Ofiolítico de Camagüey son la cromita y el olivino. En menor proporción se reconocen piroxenos (enstatita y, en menor medida, diópsido cromífero) y plagioclasa. Otras fases minoritarias son: óxidos de Ti (ilmenita y rutilo) o $\mathrm{Zr}$ (baddeleyita y un óxido de $\mathrm{Zr}$, $\mathrm{Ca}$ y $\mathrm{Ti}$ sin determinar) y sulfuros y aleaciones de $\mathrm{Fe}-\mathrm{Ni}-\mathrm{Cu}$ [pentlandita $(\mathrm{Ni}, \mathrm{Fe})_{9} \mathrm{~S}_{8}$, heazlewoodita $\left(\mathrm{Ni}_{3} \mathrm{~S}_{2}\right)$, goodlevskita $\left(\mathrm{Ni}_{9} \mathrm{~S}_{8}\right)$, calcopirita $\left(\mathrm{CuFeS}_{2}\right)$, bornita $\left(\mathrm{Cu}_{5} \mathrm{FeS}_{4}\right)$, calcocita $\left(\mathrm{CuS}_{2}\right)$, awaruita 

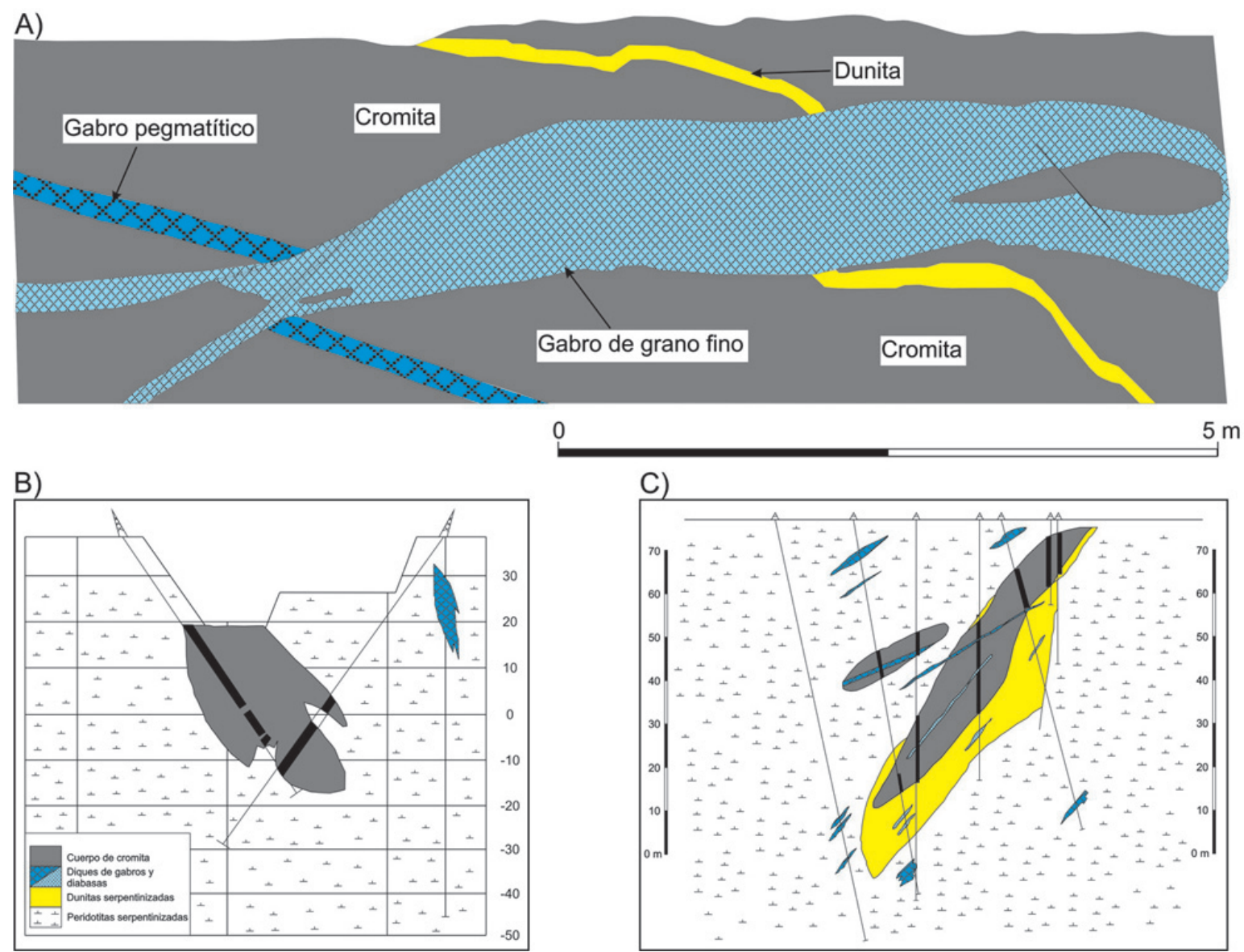

Figura 3. A) Esquema de un frente de explotación de la Mina Lolita, en Camagüey, (modificado de Flint et al., 1948). B) Corte geológico de la Mina La Mamina (modificado de González-Pontón, 2009). C) Corte geológico de la Mina Victoria (modificado de González-Pontón, 2009).

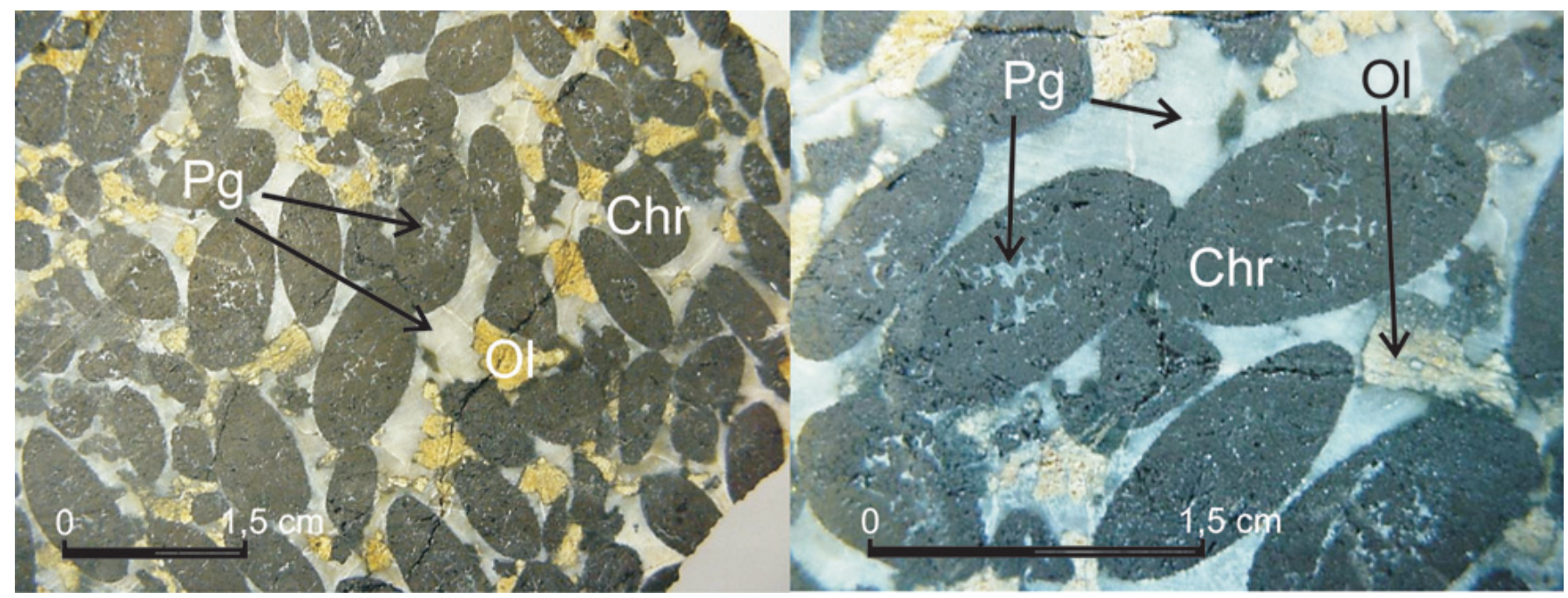

Figura 4. Ejemplo de textura nodular en una muestra de mano de la Mina Guillermina, en Camagüey. En el centro de los nódulos se observan las inclusiones de silicatos. Chr: cromita; Ol: olivino (muy alterado a serpentina); Pg: plagioclasa.

$\left(\mathrm{Ni}_{3} \mathrm{Fe}\right)$ y Cu nativo; Figura 5A, 5B, 5C, 5D, 5E, 5F, 5G, 5H y $5 \mathrm{I}]$.

Los cristales de cromita tienen, generalmente, morfologías euhedrales con tamaños de grano que no superan los $3 \mathrm{~cm}$ de longitud. En las texturas diseminadas y nodulares los cristales de cromita muestran morfologías redondeadas y/o elipsoidales (Figura 4). En ocasiones los cristales de cromita muestran estrechas bandas de alteración a ferritcromita a lo largo de los bordes de grano y fracturas abiertas (Figura 5D). El olivino $\left(\mathrm{Fo}_{95.39-91.69}\right)$, junto con la plagioclasa $\left(\mathrm{An}_{86.6-71.5}\right)$ y 
los piroxenos, forman la matriz intersticial entre los granos de cromita, aunque también pueden encontrarse, frecuentemente, como inclusiones sólidas dentro de los cristales de cromita. Cuando se encuentran en la matriz intersticial, frecuentemente están alterados a serpentina (con textura mallada), clorita y/o magnetita. Asimismo, también se reconoce granate tipo uvarovita rellenado fracturas abiertas en los cristales de cromita.

Los óxidos de Ti (ilmenita y rutilo) se presentan en forma de exsoluciones ( $25 \mu \mathrm{m}$ de longitud máxima, $20 \mu \mathrm{m}$ de anchura máxima y morfologías prismáticas, elongadas y/o equidimensionales) dentro de los cristales de cromita que forman las cromititas masivas asociadas a gabros o en algunas cromititas nodulares (Figura 5A y 5B). Estas exsoluciones se disponen, típicamente, según la orientación de los planos cristalográficos de la cromita, distribuyéndose por todo el cristal (Figura 5A y 5B). Particularmente, en torno a las exsoluciones de mayor tamaño no se desarrollan exsoluciones de tamaños menores (Figura 5B). En los cristales de cromita que forman las texturas nodulares las exsoluciones de ilmenita y rutilo pueden encontrarse, también, formando inclusiones solitarias, compuestas de rutilo e ilmenita, y/o con sulfuros de Fe-Ni-Cu [pentlandita $(\mathrm{Ni}, \mathrm{Fe})_{9} \mathrm{~S}_{8}$, godlevskita $\left(\mathrm{Ni}_{9} \mathrm{~S}_{8}\right)$, bornita $\left(\mathrm{Cu}_{5} \mathrm{FeS}_{4}\right)$ y calcopirita $\left(\mathrm{CuFeS}_{2}\right)$; Figura $\left.5 \mathrm{C}\right]$ y/o baddeleyita $\left(\mathrm{ZrO}_{2}\right)$. Cuando la ilmenita y el rutilo se asocian entre sí, dan lugar a agregados bifásicos de cristales euhedrales-subhedrales
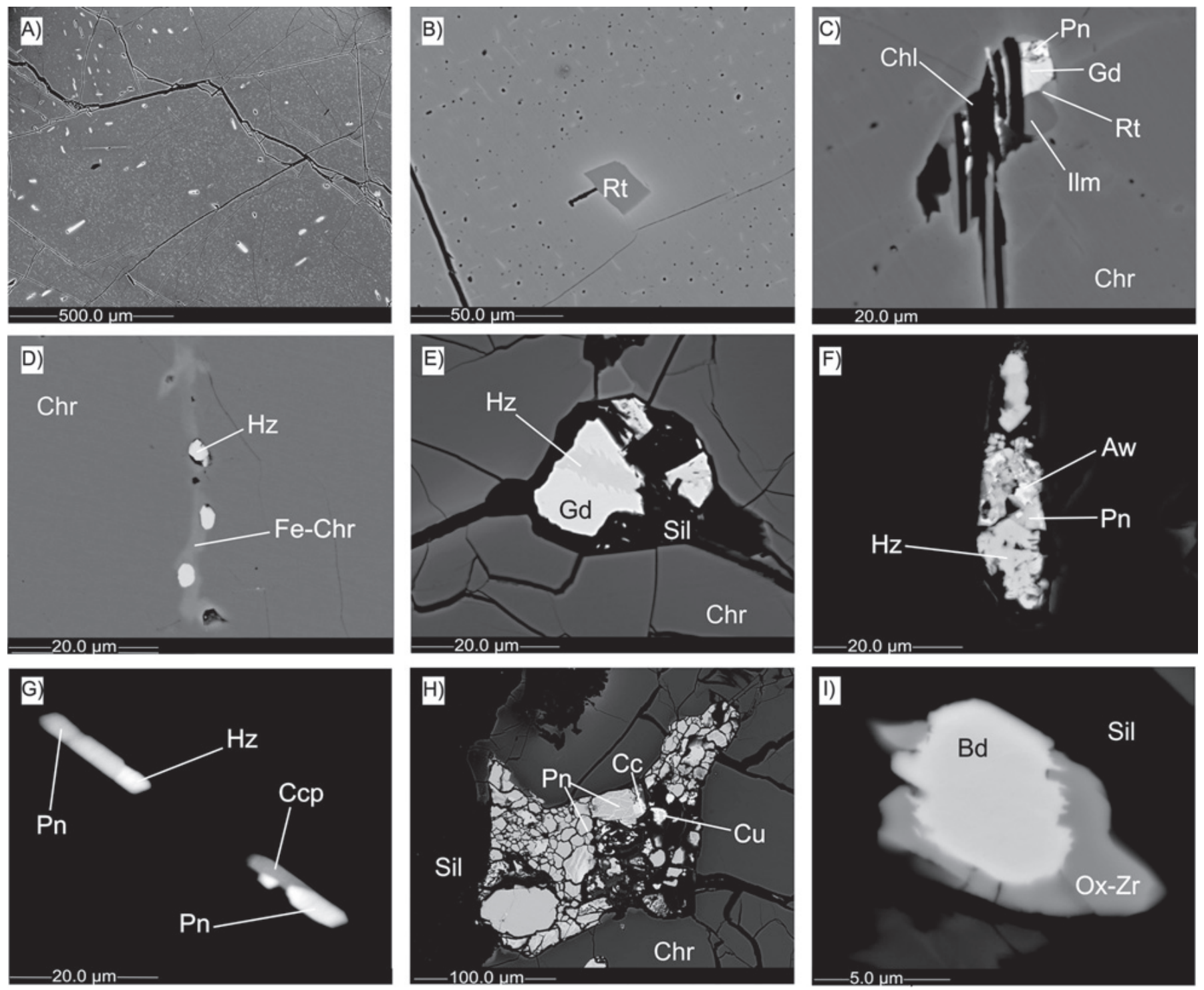

Figura 5. Imágenes de SEM representativas de las inclusiones sólidas en los cristales de cromita, en fracturas abiertas y en la matriz intersticial. A) Exsoluciones de ilmenita de diversos tamaños (color claro) en cromita (color oscuro). B) Cristal de rutilo incluido en cromita. C) Agregado de pentlandita, parcialmente alterada a godlevskita, ilmenita y rutilo en silicatos, incluidos a su vez en cromita. D) Cristales de heazlewoodita orientados en la dirección de una fractura en cromita parcialmente alterada a ferritcromita. E) Cristal de godlevskita parcialmente reemplazado por heazlewoodita en una fractura abierta en cromita. F) Cristal de pentlandita parcialmente reemplazado por awaruita y heazlewoodita en una fractura abierta en cromita. G) Agregados bifásicos de pentlandita-heazlewoodita y pentlandita-calcopirita, orientados en la dirección de una fractura. H) Agregado de pentlandita, calcocita y Cu nativo en una fractura en cromita. I) Cristal de baddeleyita parcialmente reemplazado por un óxido de $\mathrm{Zr}$ y Ca, incluido en silicatos, incluidos a su vez en cromita. Aw: awaruita; Bd: baddeleyita; Cc: calcocita; Chl: clorita; Ccp: calcopirita; Chr: cromita; $\mathrm{Cu}$ : Cu nativo; Fe-Chr: ferritcromita; Gd: godlevskita; Hz: heazlewoodita; Ilm: ilmenita; Ox-Zr: óxido de Zr; Pn: pentlandita; Rt: rutilo; Sil: silicatos. 
incluidos dentro de la cromita inalterada. Cuando se asocian con sulfuros, generalmente dan lugar a granos bifásicos o polifásicos, con hábitos subhedrales, y se encuentran incluidos en silicatos, dentro de la cromita (Figura 5C). Se reconocen también agregados de sulfuros y aleaciones de $\mathrm{Fe}-\mathrm{Ni}-\mathrm{Cu}$ [heazlewoodita $\left(\mathrm{Ni}_{3} \mathrm{~S}_{2}\right)$, goodlevskita $\left(\mathrm{Ni}_{9} \mathrm{~S}_{8}\right)$, calcocita $\left(\mathrm{CuS}_{2}\right)$, awaruita $\left(\mathrm{Ni}_{3} \mathrm{Fe}\right)$ y $\mathrm{Cu}$ nativo] y/o magnetita (Figura $5 \mathrm{D}, 5 \mathrm{E}$, $5 \mathrm{~F}, 5 \mathrm{G}$ y $5 \mathrm{H}$ ) tanto en la matriz intersticial como en fracturas abiertas en la cromita. Tales agregados se encuentran como productos de reemplazamiento de pentlandita, bornita y/o calcopirita. El óxido de Zr, Ca y Ti sin determinar reemplaza a la baddeleyita a lo largo de sus bordes de grano (Figura 5I).

\subsection{Composición química de la cromita}

La composición de la cromita de los cuerpos de cromitita del Complejo Ofiolítico de Camagüey es muy rica en $\mathrm{Al}$ (su \#Cr varía entre 0.31 y 0.6 ; Tablas 1,2 y 3). Los valores del $\# \mathrm{Mg}\left[\# \mathrm{Mg}=\mathrm{Mg} /\left(\mathrm{Mg}+\mathrm{Fe}^{2+}\right)\right]$ oscilan entre 0.35 y 0.74 y el \#Fe $\mathrm{Fe}^{3+}$ $\left[\# \mathrm{Fe}^{3+}=\mathrm{Fe}^{3+} /\left(\mathrm{Fe}^{3+}+\mathrm{Al}+\mathrm{Cr}\right)\right]$ es inferior a 0.09 . El contenido en $\mathrm{TiO}_{2}$ es relativamente elevado (hasta $1 \%$ en peso) y el de otros óxidos minoritarios es bajo: el $\mathrm{MnO}$ varía entre 0.1 y $0.43 \%$ en peso y el $\mathrm{ZnO}$ no supera el $0.19 \%$ en peso (Tablas 1,2 y $3)$.

Las muestras estudiadas de cromita se pueden dividir en dos grupos en función de su proximidad o asociación con cuerpos de gabros: las asociadas a gabros y las que no lo están. La mayoría de las muestras estudiadas no están en contacto con gabros y su \#Cr varía entre 0.31 y 0.56 (Tablas 1 y 2). Sin embargo, en las cromitas asociadas a gabros, el valor mínimo del \#Cr es 0.44 y el máximo 0.60 (Figura 6B y 6C; Tabla 3). Cabe resaltar la presencia de plagioclasa en el interior de los nódulos de cromita y en la matriz intersticial cuando el cuerpo de cromitita presenta textura nodular (Figura 4). La distribución de los cationes divalentes $\left(\mathrm{Fe}^{2+}\right.$ y $\left.\mathrm{Mg}^{2+}\right)$ muestra, igualmente, una clara diferencia: las cromitas de los cuerpos de cromitita asociados a gabros son más ricas en $\mathrm{Fe}^{2+}(\mathrm{el} \mathrm{\# Mg}$ varía entre 0.35 y 0.58 ; Figuras $6 \mathrm{~B}$ y $6 \mathrm{C}$; Tabla 3 ) que las de los cuerpos que no están en contacto con gabros (el \#Mg varía entre 0.65 y 0.74 ; Tablas 1 y 2). El contenido en $\mathrm{TiO}_{2}$ también varía entre ambos tipos de cromititas: mientras que las cromititas asociadas a gabros presentan valores intermedios entre 0.17 y $0.70 \%$ en peso de $\mathrm{TiO}_{2}$ (Tabla 3 ), aquellas que no están en contacto con gabros presentan los valores extremos de $\mathrm{TiO}_{2}$ (entre 0.04 y $1 \%$ en peso; Tablas 1 y 2 ). En el primer tipo de cromititas mencionadas anteriormente se observa un enriquecimiento progresivo en $\mathrm{TiO}_{2}$ en la cromita situada hacia el contacto con el gabro. Además, son las únicas muestras que se proyectan fuera del campo de los basaltos de dorsal mesooceánica (MORB) definido por Dick y Bullen (1984) y Arai, (1992; Figura 7A).

El diagrama del \#Cr frente al \#Mg muestra que las cromititas de Camagüey pueden clasificarse como cromititas (ofiolíticas) podiformes (Figura 6A). Esta composición llega a ser mucho más rica en $\mathrm{Al}$ que la de la cromita de otras cromititas ricas en $\mathrm{Al}$ descritas en los complejos ofiolíticos (Figura 6B y 6C). En el diagrama del \#Cr frente al contenido en TiO2 (Figura 7A) la composición de la cromita de Camagüey se proyecta, en su mayoría, dentro del campo de los basaltos de dorsal meso-oceánica (MORB; Dick y Bullen, 1984; Arai,

Tabla 1 . Análisis representativos de la composición química de la cromita de Camagüey con textura masiva que no están en contacto con gabros. n.a.: no analizado.

\begin{tabular}{|c|c|c|c|c|c|c|c|c|c|c|}
\hline Muestra & $\begin{array}{c}\text { Cu-01-1A- } \\
\text { CR-4 }\end{array}$ & $\begin{array}{c}\text { Cu-01-1A- } \\
\text { CR-4 }\end{array}$ & $\begin{array}{c}\text { Cu-01-1A- } \\
\text { CR-4 }\end{array}$ & $\begin{array}{c}\text { Cu-01-1A- } \\
\text { CR-4 }\end{array}$ & $\begin{array}{c}\text { Cu-01-06- } \\
\text { CR-3 }\end{array}$ & $\begin{array}{c}\text { Cu-01-06- } \\
\text { CR-3 }\end{array}$ & $\begin{array}{c}\text { Cu-01-06- } \\
\text { CR-3 }\end{array}$ & $\begin{array}{c}\text { Cu-01-06- } \\
\text { CR-1 }\end{array}$ & $\begin{array}{c}\text { Cu-01-06- } \\
\text { CR-1 }\end{array}$ & $\begin{array}{c}\text { Cu-01-06- } \\
\text { CR-1 }\end{array}$ \\
\hline $\mathrm{SiO}_{2}$ & n.a. & n.a. & n.a. & n.a. & n.a. & n.a. & n.a. & n.a. & n.a. & n.a. \\
\hline $\mathrm{TiO}_{2}$ & 0,32 & 0,32 & 0,32 & 0,29 & 0,39 & 0,33 & 0,22 & 0,32 & 0,33 & 0,37 \\
\hline $\mathbf{A l}_{2} \mathbf{O}_{3}$ & 25,34 & 25,08 & 25,20 & 25,42 & 25,94 & 26,47 & 26,92 & 28,26 & 28,10 & 28,19 \\
\hline $\mathrm{Cr}_{2} \mathrm{O}_{3}$ & 43,19 & 43,09 & 43,28 & 42,99 & 41,59 & 41,55 & 40,97 & 39,20 & 39,28 & 39,27 \\
\hline $\mathrm{Fe}_{2} \mathrm{O}_{3}$ & 2,99 & 3,05 & 3,39 & 2,82 & 2,83 & 2,90 & 3,32 & 3,83 & 3,38 & 3,15 \\
\hline $\mathbf{V}_{2} \mathbf{O}_{3}$ & n.a. & n.a. & n.a. & n.a. & n.a. & n.a. & n.a. & n.a. & n.a. & n.a. \\
\hline $\mathrm{FeO}$ & 12,12 & 12,28 & 12,08 & 12,11 & 12,60 & 12,26 & 11,99 & 11,76 & 11,99 & 12,24 \\
\hline MnO & 0,20 & 0,22 & 0,14 & 0,13 & 0,18 & 0,23 & 0,16 & 0,20 & 0,17 & 0,14 \\
\hline MgO & 15,64 & 15,42 & 15,76 & 15,58 & 15,22 & 15,54 & 15,75 & 16,15 & 15,89 & 15,78 \\
\hline $\mathrm{ZnO}$ & 0,03 & 0,17 & 0,04 & 0,09 & 0,10 & 0,02 & 0,06 & 0,05 & 0,02 & 0,07 \\
\hline $\mathrm{NiO}$ & n.a & n.a & n.a & n.a & n.a & n.a & $\mathrm{n} . \mathrm{a}$ & n.a & $\mathrm{n} . \mathrm{a}$ & n.a \\
\hline $\mathrm{CaO}$ & 0,00 & 0,00 & 0,00 & 0,00 & 0,00 & 0,00 & 0,00 & 0,00 & 0,00 & 0,00 \\
\hline Total & 99,82 & 99,61 & 100,22 & 99,42 & 98,85 & 99,31 & 99,41 & 99,77 & 99,15 & 99,21 \\
\hline \multicolumn{11}{|c|}{ Fórmula estructural en base a 24 oxígenos } \\
\hline $\mathbf{T i}$ & 0,01 & 0,01 & 0,01 & 0,01 & 0,01 & 0,01 & 0,01 & 0,01 & 0,01 & 0,01 \\
\hline Al & 0,75 & 0,74 & 0,74 & 0,74 & 0,76 & 0,78 & 0,79 & 0,83 & 0,83 & 0,83 \\
\hline $\mathbf{V}$ & n.a & n.a. & n.a. & n.a. & n.a. & n.a. & n.a. & n.a. & n.a. & n.a. \\
\hline $\mathrm{Cr}$ & 0,85 & 0,85 & 0,85 & 0,85 & 0,82 & 0,82 & 0,81 & 0,77 & 0,78 & 0,77 \\
\hline $\mathrm{Fe}^{3+}$ & 0,06 & 0,06 & 0,06 & 0,06 & 0,06 & 0,06 & 0,07 & 0,08 & 0,07 & 0,06 \\
\hline Mg & 0,39 & 0,38 & 0,39 & 0,39 & 0,39 & 0,39 & 0,39 & 0,40 & 0,39 & 0,39 \\
\hline Mn & 0,00 & 0,00 & 0,00 & 0,00 & 0,00 & 0,00 & 0,00 & 0,00 & 0,00 & 0,00 \\
\hline $\mathrm{Fe}^{2+}$ & 0,17 & 0,17 & 0,17 & 0,17 & 0,17 & 0,17 & 0,17 & 0,17 & 0,17 & 0,17 \\
\hline $\mathbf{N i}$ & n.a. & n.a. & n.a. & n.a. & n.a. & n.a. & n.a. & n.a. & n.a. & n.a. \\
\hline $\mathrm{Zn}$ & 0,00 & 0,00 & 0,00 & 0,00 & 0,00 & 0,00 & 0,00 & 0,00 & 0,00 & 0,00 \\
\hline$\# \mathrm{Cr}$ & 0,53 & 0,54 & 0,54 & 0,53 & 0,52 & 0,51 & 0,51 & 0,48 & 0,48 & 0,48 \\
\hline$\# \mathbf{M g}$ & 0,70 & 0,69 & 0,70 & 0,70 & 0,68 & 0,69 & 0,70 & 0,71 & 0,70 & 0,70 \\
\hline$\# \mathrm{Fe}^{3+}$ & 0,04 & 0,04 & 0,04 & 0,03 & 0,03 & 0,04 & 0,04 & 0,05 & 0,04 & 0,04 \\
\hline
\end{tabular}


Tabla 2. Análisis representativos de la composición química de la cromita de Camagüey con textura nodular (CAM-2) y diseminada (Cu-01-06-CHR) que no están en contacto con gabros. n.a.: no analizado.

\begin{tabular}{|c|c|c|c|c|c|c|c|c|c|c|}
\hline Muestra & CAM-2 & CAM-2 & CAM-2 & CAM-2 & CAM-2 & $\begin{array}{c}\text { Cu-01-06- } \\
\text { CHR }\end{array}$ & $\begin{array}{c}\text { Cu-01-06- } \\
\text { CHR }\end{array}$ & $\begin{array}{c}\text { Cu-01-06- } \\
\text { CHR }\end{array}$ & $\begin{array}{c}\mathrm{Cu-01-06-} \\
\mathrm{CHR}\end{array}$ & $\begin{array}{c}\text { Cu-01-06- } \\
\text { CHR }\end{array}$ \\
\hline $\mathrm{SiO}_{2}$ & n.a. & n.a. & n.a. & n.a. & n.a. & n.a. & n.a. & n.a. & n.a. & n.a. \\
\hline $\mathrm{TiO}_{2}$ & 0,59 & 0,58 & 0,57 & 0,61 & 0,55 & 0,29 & 0,32 & 0,32 & 0,32 & 0,31 \\
\hline $\mathbf{A l}_{2} \mathbf{O}_{3}$ & 29,97 & 30,00 & 29,82 & 29,83 & 30,16 & 36,09 & 36,16 & 35,55 & 36,28 & 37,28 \\
\hline $\mathrm{Cr}_{2} \mathrm{O}_{3}$ & 36,89 & 37,01 & 36,95 & 36,78 & 36,77 & 32,17 & 32,29 & 31,60 & 32,07 & 31,02 \\
\hline $\mathrm{Fe}_{2} \mathrm{O}_{3}$ & 3,76 & 3,80 & 3,58 & 3,50 & 3,50 & 2,15 & 2,31 & 3,37 & 2,25 & 2,01 \\
\hline $\mathbf{V}_{2} \mathbf{O}_{3}$ & n.a. & n.a. & n.a. & n.a. & n.a. & n.a. & n.a. & n.a. & n.a. & n.a. \\
\hline $\mathrm{FeO}$ & 12,33 & 12,34 & 12,59 & 12,25 & 12,10 & 12,19 & 12,48 & 11,66 & 12,26 & 12,44 \\
\hline MnO & 0,25 & 0,14 & 0,20 & 0,17 & 0,21 & 0,12 & 0,16 & 0,21 & 0,18 & 0,19 \\
\hline MgO & 16,11 & 16,21 & 15,90 & 16,07 & 16,21 & 16,73 & 16,67 & 16,94 & 16,75 & 16,70 \\
\hline $\mathrm{ZnO}$ & 0,02 & 0,07 & 0,05 & 0,06 & 0,11 & 0,09 & 0,08 & 0,08 & 0,05 & 0,16 \\
\hline $\mathrm{NiO}$ & n.a. & n.a. & n.a. & n.a. & n.a. & n.a. & n.a. & n.a. & n.a. & n.a. \\
\hline $\mathrm{CaO}$ & 0,00 & 0,02 & 0,01 & 0,00 & 0,00 & 0,01 & 0,01 & 0,04 & 0,00 & 0,00 \\
\hline Total & 99,91 & 100,16 & 99,68 & 99,27 & 99,61 & 99,83 & 100,48 & 99,76 & 100,17 & 100,13 \\
\hline \multicolumn{11}{|c|}{ Fórmula estructural en base a 24 oxígenos } \\
\hline $\mathbf{T i}$ & 0,01 & 0,01 & 0,01 & 0,02 & 0,01 & 0,01 & 0,01 & 0,01 & 0,01 & 0,01 \\
\hline Al & 0,88 & 0,88 & 0,88 & 0,88 & 0,89 & 1,06 & 1,06 & 1,05 & 1,07 & 1,10 \\
\hline $\mathbf{V}$ & n.a. & n.a. & n.a. & n.a. & n.a. & n.a. & n.a. & n.a. & n.a. & n.a. \\
\hline $\mathrm{Cr}$ & 0,73 & 0,73 & 0,73 & 0,73 & 0,73 & 0,63 & 0,64 & 0,62 & 0,63 & 0,61 \\
\hline $\mathrm{Fe}^{3+}$ & 0,08 & 0,08 & 0,07 & 0,07 & 0,07 & 0,04 & 0,05 & 0,07 & 0,05 & 0,04 \\
\hline Mg & 0,40 & 0,40 & 0,39 & 0,40 & 0,40 & 0,41 & 0,41 & 0,42 & 0,42 & 0,41 \\
\hline Mn & 0,00 & 0,00 & 0,00 & 0,00 & 0,00 & 0,00 & 0,00 & 0,00 & 0,00 & 0,00 \\
\hline $\mathrm{Fe}^{2+}$ & 0,17 & 0,17 & 0,18 & 0,17 & 0,17 & 0,17 & 0,17 & 0,16 & 0,17 & 0,17 \\
\hline $\mathbf{N i}$ & n.a. & n.a. & n.a. & n.a. & n.a. & n.a. & n.a. & n.a. & n.a. & n.a. \\
\hline $\mathbf{Z n}$ & 0,00 & 0,00 & 0,00 & 0,00 & 0,00 & 0,00 & 0,00 & 0,00 & 0,00 & 0,00 \\
\hline$\# \mathbf{C r}$ & 0,45 & 0,45 & 0,45 & 0,45 & 0,45 & 0,37 & 0,37 & 0,37 & 0,37 & 0,36 \\
\hline$\# \mathbf{M g}$ & 0,70 & 0,70 & 0,69 & 0,70 & 0,70 & 0,71 & 0,70 & 0,72 & 0,71 & 0,71 \\
\hline$\# \mathrm{Fe}^{3+}$ & 0,04 & 0,05 & 0,04 & 0,04 & 0,04 & 0,02 & 0,03 & 0,04 & 0,03 & 0,02 \\
\hline
\end{tabular}

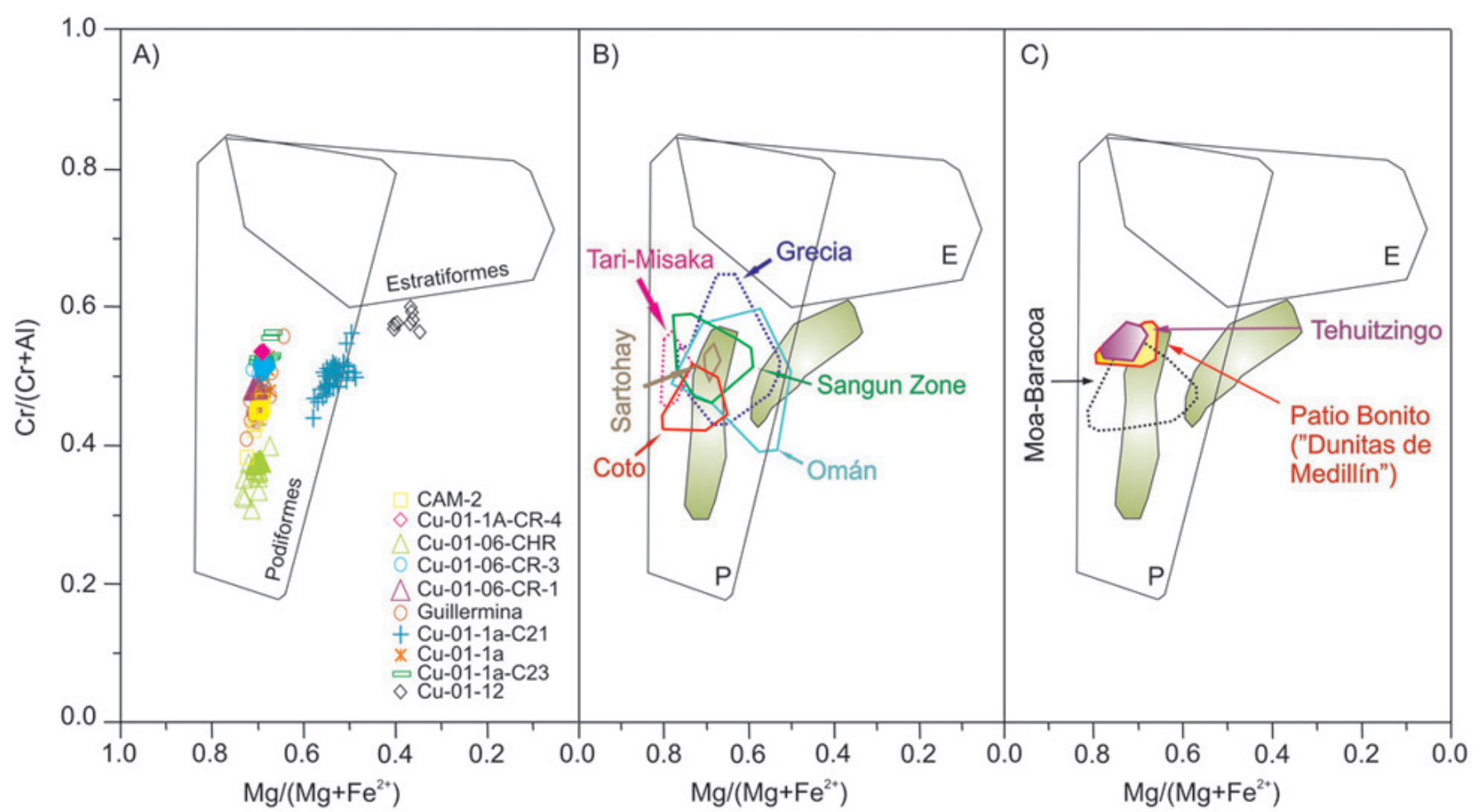

Figura 6. Diagrama del \#Cr frente al \#Mg de la cromita de Camagüey y de la cromita rica en Al de otros complejos ofiolíticos. A) Complejo Ofiolítico de Camagüey, Cuba Central. B) Cromitas ricas en Al de: Omán (Augé, 1987; Ahmed y Arai, 2002), Grecia (Economou-Eliopoulos, 1986, 1993; EconomouEliopoulos, 1995; Garuti et al., 1999), Sangun Zone (Matsumoto et al., 1997), Tari-Misaka (Arai y Yurimoto, 1994), Sartohay (Zhou et al., 2001) y Coto (Zhou et al., 2000). C) Cromita rica en Al de algunos complejos de América Latina: Moa-Baracoa (Proenza et al., 1999b), Tehuitzingo (Proenza et al., 2004a; Zaccarini et al., 2005), Patio Bonito (Proenza et al., 2004b). Campo verde izquierdo (en B y C): cromita de Camagüey no asociada a cuerpos de gabros; campo verde derecho (en $\mathrm{B}$ y C): cromita de Camagüey asociada a gabros. 
Tabla 3. Análisis representativos de la composición química de la cromita de Camagüey con textura masiva que están en contacto con gabros. n.a.: no analzado.

\begin{tabular}{|c|c|c|c|c|c|c|c|c|c|c|}
\hline Muestra & $\begin{array}{c}\text { Cu-01-1a- } \\
\text { C21 }\end{array}$ & $\begin{array}{c}\text { Cu-01-1a- } \\
\text { C21 }\end{array}$ & $\begin{array}{l}\text { Cu-01-1a- } \\
\text { C21 }\end{array}$ & $\begin{array}{c}\text { Cu-01-1a- } \\
\text { C21 }\end{array}$ & $\begin{array}{l}\text { Cu-01-1a- } \\
\text { C21 }\end{array}$ & $\mathrm{Cu}-01-12$ & $\mathrm{Cu}-01-12$ & Cu-01-12 & $\mathrm{Cu}-01-12$ & $\mathrm{Cu}-01-1$ \\
\hline $\mathrm{SiO}_{2}$ & 0,03 & 0,02 & 0,01 & 0,03 & 0,01 & n.a. & n.a. & n.a. & n.a. & n.a. \\
\hline $\mathrm{TiO}_{2}$ & 0,69 & 0,20 & 0,10 & 0,48 & 0,07 & 1,00 & 0,96 & 0,99 & 0,96 & 1,00 \\
\hline $\mathbf{A l}_{2} \mathbf{O}_{3}$ & 24,23 & 24,93 & 29,61 & 25,90 & 31,11 & 19,60 & 19,87 & 20,14 & 19,09 & 20,11 \\
\hline $\mathrm{Cr}_{2} \mathrm{O}_{3}$ & 38,75 & 38,07 & 37,97 & 36,95 & 36,28 & 40,70 & 40,31 & 40,63 & 41,41 & 40,88 \\
\hline $\mathrm{Fe}_{2} \mathrm{O}_{3}$ & 5,81 & 6,59 & 1,98 & 6,38 & 2,09 & 6,59 & 6,82 & 6,49 & 6,35 & 6,38 \\
\hline $\mathbf{V}_{2} \mathbf{O}_{3}$ & 0,25 & 0,26 & 0,35 & 0,21 & 0,26 & n.a. & n.a. & n.a. & n.a. & n.a. \\
\hline $\mathrm{FeO}$ & 18,06 & 17,93 & 17,29 & 17,96 & 16,84 & 23,81 & 23,57 & 22,41 & 23,61 & 22,72 \\
\hline MnO & 0,34 & 0,34 & 0,28 & 0,36 & 0,34 & 0,42 & 0,43 & 0,40 & 0,39 & 0,42 \\
\hline MgO & 11,70 & 11,61 & 12,70 & 11,91 & 12,96 & 7,62 & 7,77 & 8,57 & 7,63 & 8,42 \\
\hline $\mathrm{ZnO}$ & n.a. & n.a. & n.a. & n.a. & n.a. & 0,16 & 0,15 & 0,09 & 0,17 & 0,12 \\
\hline $\mathrm{NiO}$ & 0,25 & 0,19 & 0,03 & 0,12 & 0,13 & n.a. & n.a. & n.a. & n.a. & n.a. \\
\hline $\mathrm{CaO}$ & n.a. & n.a. & n.a. & n.a. & n.a. & 0,02 & 0,02 & 0,02 & 0,03 & 0,01 \\
\hline Total & 100,11 & 100,14 & 100,32 & 100,30 & 100,09 & 100,20 & 100,19 & 100,06 & 99,92 & 100,36 \\
\hline $\mathbf{T i}$ & 0,02 & 0,01 & 0,00 & 0,01 & 0,00 & 0,03 & 0,02 & 0,02 & 0,02 & 0,02 \\
\hline Al & 0,88 & 0,90 & 0,87 & 0,92 & 1,09 & 0,58 & 0,58 & 0,59 & 0,56 & 0,59 \\
\hline V & 0,00 & 0,00 & 0,01 & 0,00 & 0,00 & n.a. & n.a. & n.a. & n.a. & n.a. \\
\hline $\mathrm{Cr}$ & 0,95 & 0,93 & 1,11 & 0,92 & 0,85 & 0,80 & 0,80 & 0,80 & 0,82 & 0,81 \\
\hline $\mathrm{Fe}^{3+}$ & 0,14 & 0,15 & 0,01 & 0,14 & 0,05 & 0,13 & 0,14 & 0,13 & 0,13 & 0,13 \\
\hline Mg & 0,54 & 0,53 & 0,49 & 0,53 & 0,57 & 0,19 & 0,19 & 0,21 & 0,19 & 0,21 \\
\hline Mn & 0,01 & 0,01 & 0,01 & 0,01 & 0,01 & 0,01 & 0,01 & 0,01 & 0,01 & 0,01 \\
\hline $\mathrm{Fe}^{2+}$ & 0,47 & 0,46 & 0,50 & 0,47 & 0,42 & 0,33 & 0,33 & 0,32 & 0,33 & 0,32 \\
\hline $\mathbf{N i}$ & 0,01 & 0,01 & 0,00 & 0,00 & 0,00 & n.a. & n.a. & n.a. & n.a. & n.a. \\
\hline $\mathrm{Zn}$ & 0,00 & 0,00 & 0,00 & 0,00 & 0,00 & 0,00 & 0,00 & 0,00 & 0,00 & 0,00 \\
\hline$\# \mathbf{C r}$ & 0,52 & 0,51 & 0,46 & 0,49 & 0,44 & 0,58 & 0,58 & 0,58 & 0,59 & 0,58 \\
\hline$\# \mathbf{M g}$ & 0,54 & 0,54 & 0,57 & 0,54 & 0,58 & 0,36 & 0,37 & 0,40 & 0,36 & 0,40 \\
\hline$\# \mathbf{F e}^{3+}$ & 0,07 & 0,08 & 0,03 & 0,08 & 0,02 & 0,09 & 0,09 & 0,09 & 0,08 & 0,08 \\
\hline
\end{tabular}

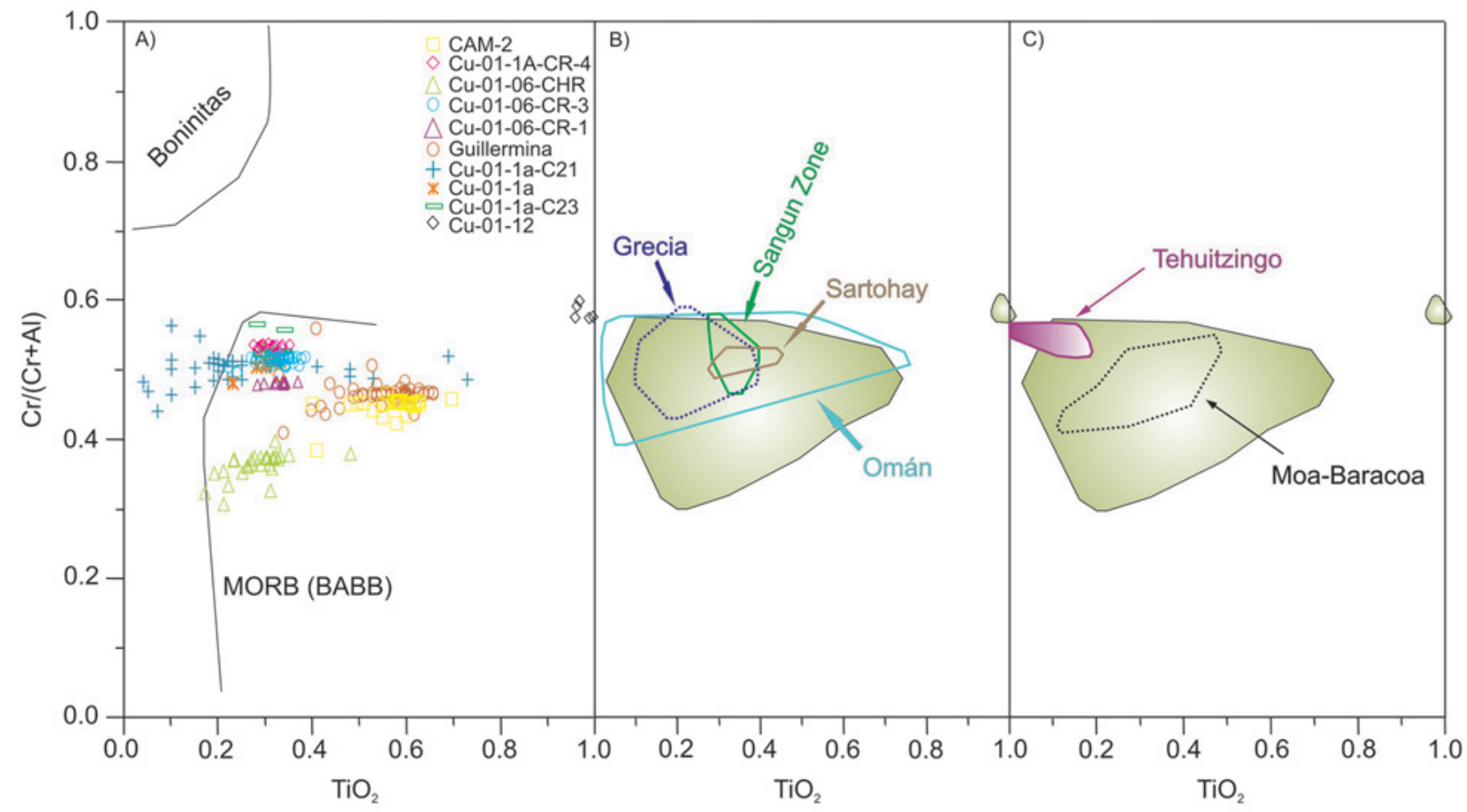

Figura 7. Diagrama del \#Cr frente al contenido en TiO2 de la cromita de Camagüey y de la cromita rica en Al de otros complejos ofiolíticos. A) Complejo Ofiolítico de Camagüey. B) Cromita rica en Al de: Omán (Augé, 1987; Ahmed y Arai, 2002), Grecia (Economou-Eliopoulos, 1986, 1993; Economou-Eliopoulos, 1995; Garuti et al., 1999), Sangun Zone (Matsumoto et al., 1997), Sartohay (Zhou et al., 2001). C) Cromita rica en Al de algunos complejos de América Latina: Moa-Baracoa (Proenza et al., 1999b) y Tehuitzingo (Proenza et al., 2004a; Zaccarini et al., 2005). Campos verdes en B y C: cromita de Camagüey. 


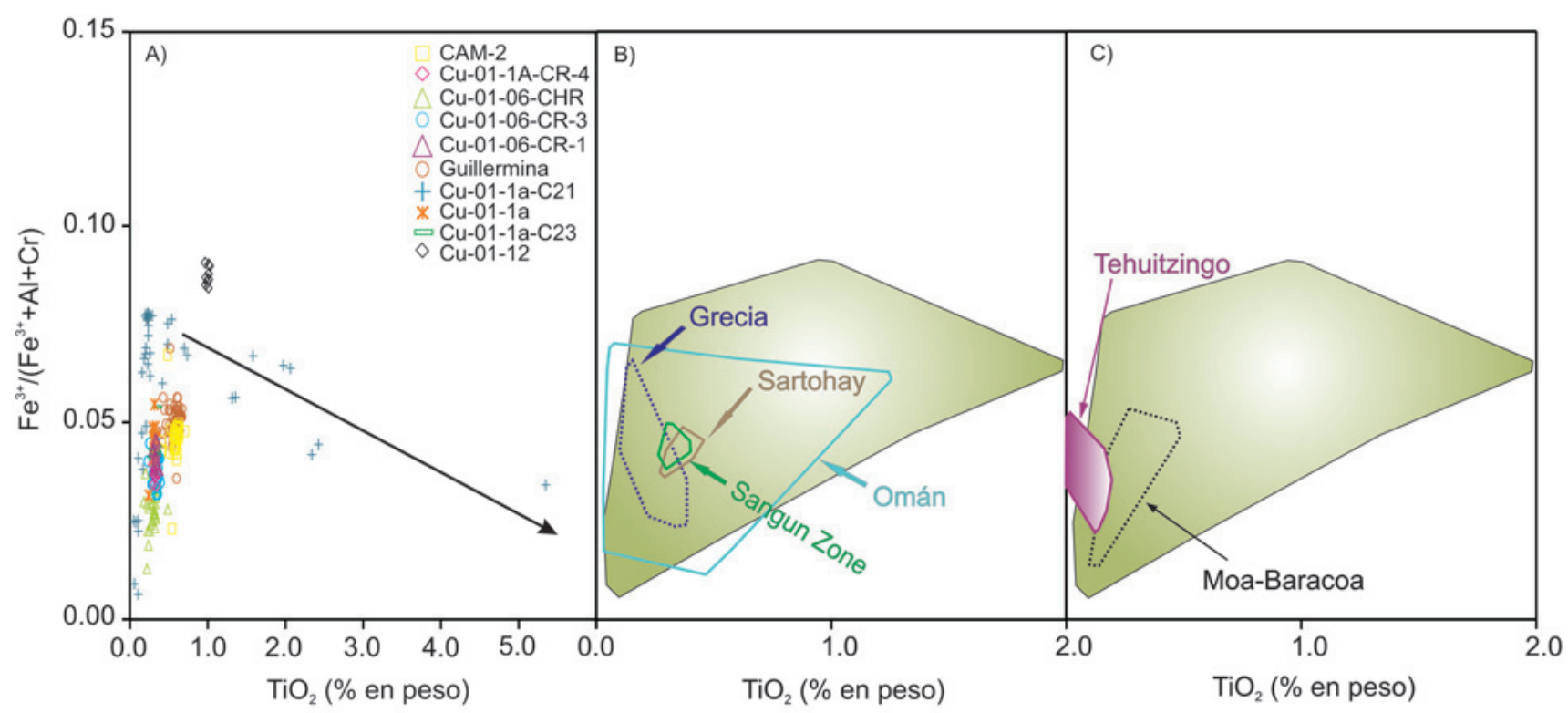

Figura 8. Diagrama del $\# \mathrm{Fe}^{3+}$ frente al contenido en $\mathrm{TiO}_{2}$ de la cromita de Camagüey y de la cromita rica en $\mathrm{Al}$ de otros complejos ofiolíticos. A) Complejo Ofiolítico de Camagüey; flecha: tendencia mostrada por los análisis de cromita contaminados por exsoluciones de ilmenita. B) Cromita rica en Al de: Omán (Augé, 1987; Ahmed y Arai, 2002), Grecia (Economou-Eliopoulos, 1986, 1993; Economou-Eliopoulos, 1995; Garuti et al., 1999), Sangun Zone (Matsumoto et al., 1997) y Sartohay (Zhou et al., 2001). C) Cromita rica en Al de algunos complejos de América Latina: Moa-Baracoa (Proenza et al., 1999b) y Tehuitzingo (Proenza et al., 2004a; Zaccarini et al., 2005). Campo verde en B y C: cromita de Camagüey.

1992). Esta composición presenta valores del contenido en $\mathrm{TiO}_{2}$ muy superiores a los que muestran otras cromititas ofiolíticas ricas en Al (Figuras 7B y 7C). En las cromititas del Complejo Ofiolítico de Camagüey el $\# \mathrm{Fe}^{3+}$ y el contenido en $\mathrm{TiO}_{2}$ se correlacionan positivamente de manera similar a lo que ocurre en otros complejos ofiolíticos (Figuras 8A, 8B, $8 \mathrm{C})$. No obstante, algunas muestras de cromitita en contacto con gabros, muestran una tendencia diferente (Figura 8A). Esto se debe a que en esos puntos, los análisis realizados sobre cromita están contaminados por exsoluciones de ilmenita tal y como demuestra el hecho de que los altos contenidos de $\mathrm{TiO}_{2}$ se relacionan con bajos contenidos de $\mathrm{Fe}^{3+}$ y de $\mathrm{Mg}$, elementos estos que no están presentes en el rutilo o la ilmenita.

$\mathrm{Al}$ igual que ocurre en otras cromititas ricas en $\mathrm{Al}$ de la Faja Ofiolítica de Mayarí-Baracoa (Proenza et al., 1999a, 1999b), en las cromititas de Camagüey el contenido en $\mathrm{Cr}$ de la cromita que forma los cuerpos de cromitita es menor que el que presenta la cromita accesoria de la dunita y de la harzburgita encajantes, respectivamente (Figura 9). Esta tendencia, sin embargo, es contraria a la que se observa en el caso del $\mathrm{TiO}_{2}$ y, por tanto, del Al (Figura 9), los cuales se incrementan a medida que disminuye el contenido en $\mathrm{Cr}$.

\section{Discusión}

Las cromititas del Complejo Ofiolítico de Camagüey presentan una serie de características petrológicas y químicas que, por un lado, subrayan su naturaleza de cromititas ricas en $\mathrm{Al} \mathrm{y}$, por otro, las diferencian de las descritas hasta ahora en la literatura. Las cromititas se localizan en la zona de transición manto-corteza y están cortadas por abundantes diques de gabros y/o troctolitas (como ocurre, por ejemplo, en el macizo de Moa-Baracoa, en Cuba oriental; Proenza et al., 1999a, 1999b). Sin embargo, frecuentemente las cromititas contienen plagioclasa primaria en el interior de los nódulos de cromita y, sobre todo, en la matriz intersticial. Asimismo, la composición de la cromita llega a ser mucho más rica en $\mathrm{Al}$ (hasta \#Cr=0.31) que la descrita en otras cromititas ofiolíticas ricas en Al (Augé, 1987; Economou-Eliopoulos, 1986, 1993; Economou-Eliopoulos, 1995; Arai y Yurimoto, 1994; Matsumoto et al., 1997; Garuti et al., 1999; Proenza et al., 1999b, 2004a, 2004b; Zhou et al., 2000; Zhou et al., 2001; Ahmed y Arai, 2002). Las cromititas de Camagüey presentan contenidos de Ti relativamente elevados (de hasta un $1 \%$ en peso) y abundantes exsoluciones de ilmenita y/o rutilo, lo que pone de manifiesto que el contenido original (previo a la exsolución) fue aún mayor. En el caso de las cromititas en contacto directo con gabros el contenido de $\mathrm{TiO}_{2}$ de la cromita aumenta hacia dicho contacto.

\subsection{Origen de las cromititas ricas en Al del Complejo Ofiolítico de Camagüey}

La disposición discordante de las rocas feldespáticas en relación con los cuerpos de cromititas sugiere un origen previo de las cromititas (Figura 3A). De acuerdo con Zhou et al. (2001) dichas relaciones entre cromititas ricas en $\mathrm{Al}$ y rocas feldespáticas, que se reconocen en Sartohay, en el Complejo Ofiolítico de Dalabute (China), son el resultado de la reacción entre fundidos toleíticos pobres en $\mathrm{Si}$ y ricos en $\mathrm{Al}_{2} \mathrm{O}_{3}$ y las peridotitas mantélicas. La reacción fundido/peridotita da lugar a la disolución selectiva del piroxeno de la peridotita favoreciendo la cristalización de olivino y cromita. En este modelo la cristalización de cromita tendría lugar en los canales de percolación magmática representados por las dunitas encajantes (Zhou et al., 1994; Proenza et al., 1999a, 1999b). 

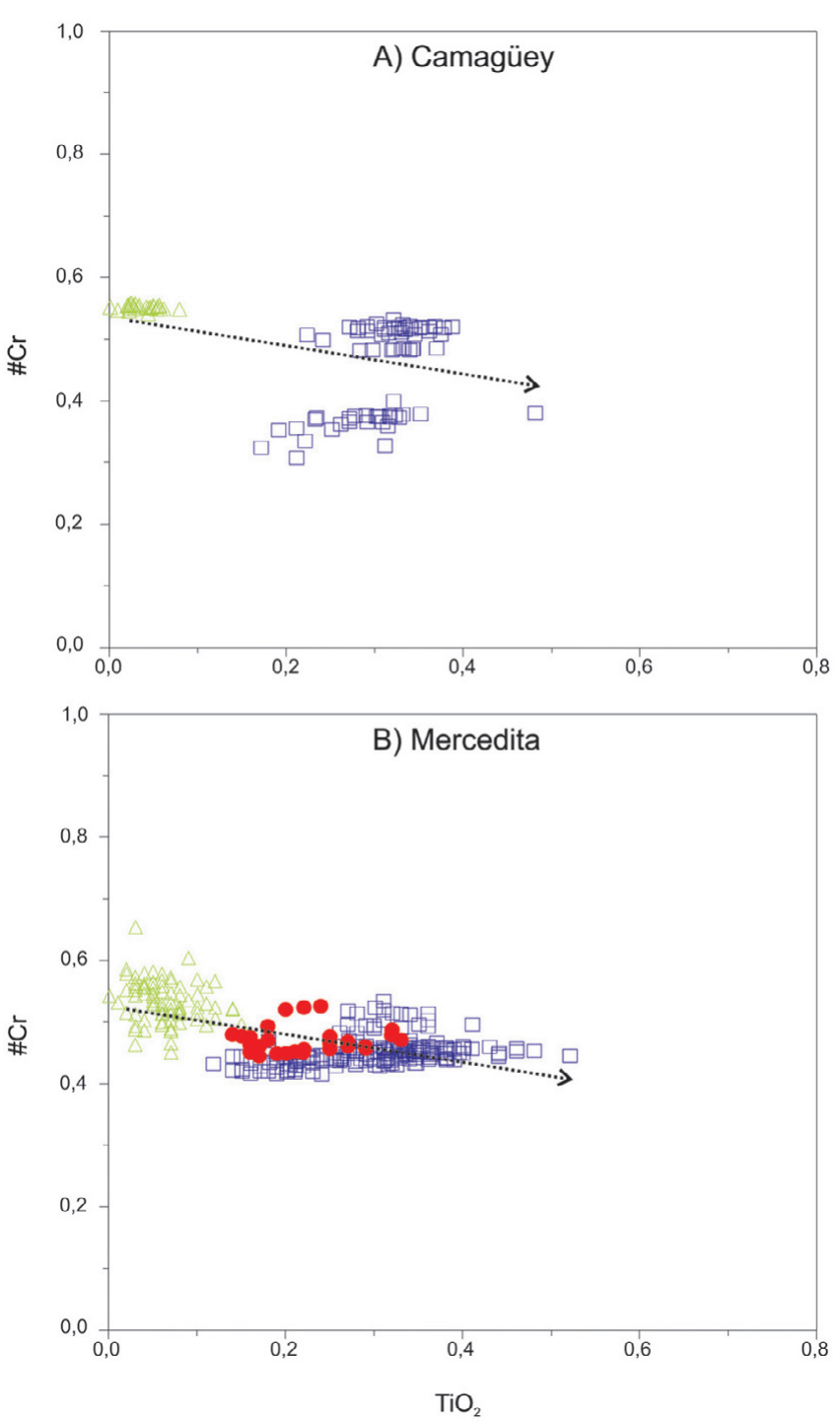

Figura 9. Diagramas del \#Cr frente al contenido en $\mathrm{TiO}_{2}$ de la cromita rica en Al de: A) Camagüey y B) Mercedita, distrito de Moa-Baracoa, Cuba Oriental. Triángulos verdes: cromita accesoria en las harzburgitas; círculos rojos: cromita accesoria en las dunitas; cuadrados azules: cromita de los cuerpos de cromitita; flechas: tendencia mostrada por los análisis de cromita en harzburgitas, dunitas y cromititas.

Durante el enfriamiento del bloque litosférico, los fundidos remanentes con bajas relaciones $\mathrm{Ca} / \mathrm{Al}$ cristalizarían dando lugar a los diques de rocas feldespáticas (gabros y troctolitas) que intruirían tanto a las cromititas como a las peridotitas encajantes. Sin embargo, el mayor contenido en $\mathrm{TiO}_{2}$ en la cromitita justo en el contacto con algunos gabros junto con el hecho de que, frecuentemente, los nódulos de cromita o la matriz de la cromitita contengan plagioclasa primaria hace pensar (de acuerdo con las tendencias descritas por Proenza et al., 1999b, en las cromititas del Complejo de Moa-Baracoa) que estos gabros podrían haber sido originalmente, sills asimilados durante la formación de la cromitita (Bédard y Hébert, 1998). Además, estos cuerpos de gabro son muy abundantes en las zonas más próximas a la corteza (Figura 2). La adición de $\mathrm{SiO}_{2}$, $\mathrm{Al}_{2} \mathrm{O}_{3}$ y volátiles al magma tras el reemplazamiento de gabros y troctolitas explicaría la tendencia de disminución del \#Cr e incremento del contenido en $\mathrm{TiO}_{2}$ en la cromita accesoria de la harzburgita, la dunita y la cromitita, respectivamente, lo que sugiere un fuerte desequilibrio químico entre los fundidos que dieron lugar a las cromititas y los fundidos en equilibrio con las peridotitas encajantes.

La abundancia de exsoluciones e inclusiones de ilmenita y/o rutilo primarias en los cristales de cromita sugiere que el contenido en Ti del magma parental de la cromitita fue tan elevado que no pudo ser albergado en la estructura de la cromita.

\subsection{Procesos de alteración de las cromititas}

En las cromititas del Complejo Ofiolítico de Camagüey y en las peridotitas encajantes el olivino y los piroxenos están frecuentemente reemplazados por serpentina. Las asociaciones de minerales de metales base que se reconocen en las zonas serpentinizadas están formadas por sulfuros de $\mathrm{Ni}$ pobres en S (heazlewoodita, godlevskita), aleaciones (awaruita) y óxidos (magnetita) que reemplazan a la pentlandita primaria y calcocita o $\mathrm{Cu}$ nativo que alteran a la bornita o a la calcopirita primarias.

Estas asociaciones de minerales secundarios sugieren procesos de serpentinización muy probablemente en un contexto de fondo oceánico (Eckstrand, 1975; Frost, 1985; Klein y Bach, 2009). La formación de serpentina, brucita y magnetita como producto de alteración del olivino tiene lugar bajo condiciones de baja $f \mathrm{~S}_{2}$ y $f \mathrm{O}_{2}$ en el medio (Frost, 1985; Bach et al., 2006). En este contexto altamente reductor el $\mathrm{H}_{2}$ liberado reacciona con el $\mathrm{O}_{2}$ para formar agua y con el $\mathrm{S}_{2}$ para formar $\mathrm{H}_{2} \mathrm{~S}$. En tales condiciones, los sulfuros primarios no son estables y tienden a equilibrarse perdiendo $\mathrm{S}$ y dando lugar a sulfuros más pobres en $\mathrm{S}$ o a aleaciones (Eckstrand, 1975; Frost, 1985). Así, en las cromititas de Camagüey, la pentlandita está reemplazada por heazlewoodita, godlevskita y/o awaruita. La formación local de estrechos bordes de alteración de ferritcromita, clorita, magnetita y/o granate tipo uvarovita rellenando fracturas se asocia con etapas más tardías de serpentinización, a mayores fugacidades de oxígeno (Proenza et al., 1999c).

\section{Conclusiones}

(1) En el Complejo Ofiolítico de Camagüey se reconocen numerosos cuerpos podiformes de cromitita. La composición de las cromititas es las más rica en $\mathrm{Al}$ de entre todas las cromititas ofiolíticas descritas en la literatura. Estas cromititas se localizan en una MTZ dominada por harzburgitas, dunitas y rocas feldespáticas, las cuales presentan diferentes relaciones de concordancia y discordancia con los cuerpos de cromitita.

(2) Las cromititas del Complejo Ofiolítico de Camagüey muestran contenidos relativamente elevados de Ti (hasta un $1 \%$ en peso de $\mathrm{TiO}_{2}$ ). Además, este contenido fue originalmente mayor (a alta temperatura), como se pone de manifiesto por la presencia de abundantes exsoluciones lamelares de ilmenita y rutilo orientadas paralelamente a los planos cristalográficos de la cromita. 
(3) Las características petrológicas y químicas de las cromititas sugieren que éstas se formaron a partir de reacciones fundido/peridotita o por reemplazamiento de rocas feldespáticas (gabros y/o troctolitas) preexistentes.

(4) El proceso de alteración predominante que afectó a las cromititas fue la serpentinización de fondo oceánico. Durante dicho proceso, la cromita se alteró, ligeramente, a ferritcromita y los silicatos primarios de la ganga (olivino, piroxeno y, en menor medida, plagioclasa) fueron reemplazados por silicatos secundarios tales como serpentina, brucita, magnetita y, en menores proporciones, clorita y granate tipo uvarovita. Las bajas condiciones de $f \mathrm{O}_{2}$ y $f \mathrm{~S}_{2}$ generadas durante los estadios iniciales de la serpentinización conllevaron la alteración de los sulfuros primarios, dando lugar a otros sulfuros más pobres en $\mathrm{S}$ y/o a aleaciones.

\section{Agradecimientos}

Los autores de este trabajo queremos mostrar nuestra gratitud a Jesús Montes Rueda (Departamento de Mineralogía y Petrología) por su esmero en la preparación de las secciones delgado pulidas de las muestras de cromititas. Así como al Dr. John Lewis (George Washington University) por cedernos algunas de las muestras de cromititas estudiadas. También agradecemos el especial apoyo brindando por Isabel Sánchez Almazo (CEAMA, Junta de Andalucía-Universidad de Granada), Miguel Ángel Hidalgo Laguna (Centro de Instrumentación Científica, Universidad de Granada) y Xavier Llovet (Serveis Cientificotécnics, Universitat de Barcelona) durante los trabajos con ESEM y microsonda electrónica, respectivamente. Este trabajo se costeó con fondos procedentes de los proyectos CGL2007-61205 y CGL2006-07384 del Ministerio de Ciencia y Tecnología de España, y de los grupos de investigación RNM131 de la Junta de Andalucía y 2009_ SGR444 de la Generalitat de Catalunya.

\section{Referencias}

Ahmed, A.H., Arai, S., 2002, Unexpectedly high-PGE chromitite from the deeper mantle section of the northern Oman ophiolite and its tectonics implications: Contributions to Mineralogy and Petrology, 143, 263-278.

Ahmed, A.H., Arai, S., 2003, Platinum-group minerals in podiforms chromitites of the Oman ophiolite: Canadian Mineralogist, 41, 597-616.

Albear, J.F., Iturralde-Vinent, M.A., 1985, Tectonic setting of the gabbroperidotite complex of Havana provinces, en Iturralde-Vinent, M. (ed.) Contribución a la Geología de las Provincias de La Habana y Ciudad de la Habana: La Habana, Cuba, Científico-Técnica, 87-93.

Andó, J., Harangi, S., Szakmány, B., Osztály, L., 1996, Petrología de la asociación ofiolítica de Holguín, en Iturralde-Vinent, M.A. (ed.), Ofiolitas y arcos volcánicos de Cuba: Miami, E.U.A., First Contribution IGCP Project 364, 154-176.

Arai, S., 1992, Chemistry of chromian spinel in volcanic rocks as a potential guide to magma chemistry: Mineralogical Magazine, 56, 173-184.

Arai, S., Yurimoto, H., 1994, Podiform chromitites of the Tari-Misaka ultramafic complex, Southwestern Japan, as mantle-melt interaction products: Economic Geology, 89, 1279-1288.

Augé, T., 1987, Chromite deposits in the Northern Oman ophiolite: mineralogical constraints: Mineralium Deposita, 22, 1-10.

Bach, W., Paulick, H., Garrido, C.J., Ildefonse, B., Meurer, W., Humphris, S.E., 2006, Unravelling the sequence of serpentinization reactions: petrography, mineral chemistry, and petrophysics of serpentinites from
MAR $15^{\circ} \mathrm{N}$ (ODP Leg 209, Site 1274): Geophysical Research Letters, 33, L13306.

Bédard, J.H., Hébert, R., 1998, Formation of chromitites by assimilation of crustal pyroxenites and gabbros into peridotitic intrusions: North Arm Mountain massif, Bay of Islands ophiolite, Newfoundland, Canada: Journal of Geophysical Research, 103, 5165-5184.

Carmichael, I.S.E., 1967, The mineralogy and petrology of the volcanic rocks from the Leucite Hills, Wyoming: Contributions to Mineralogy and Petrology, 15, 24-66.

Dick, H.J.B., Bullen, T., 1984, Chromian spinel as a petrogenetic indicator in abyssal and alpine-type peridotites and spatially associated lavas: Contributions to Mineralogy and Petrology, 86, 54-76.

Eckstrand, O.D., 1975, The Dumont serpentinite: a model for control of nickeliferous opaque mineral assemblages by alteration reactions in ultramafic rocks: Economic Geology, 70, 183-201.

Economou-Eliopoulos, M., 1986, Platinum-Group Elements (PGE) in chromite and sulphide ores within the ultramafic zone of some Greek ophiolite complexes, in Gallagher, M.J. et al. (eds.), Metallogeny of basic and ultrabasic rocks: London, Institution of Mining and Metallurgy, 441-454.

Economou-Eliopoulos, M., 1993, Platinum-group elements (PGE) distribution in chromite ores from ophiolite complexes of Greece: implications for exploration: Ophioliti, 18, 83-97.

Economou-Eliopoulos, M., Vacondios, I., 1995, Geochemistry of chromitites and host rocks from the Pindos ophiolite complex, northwestern Greece: Chemical Geology, 122, 99-108.

Flint, D.E., Albear, J.F., Guild, P.W., 1948, Geology and chromite deposits of the Camagüey District, Camagüey Province, Cuba: U.S. Geological Survey Bulletin, 954, 39-63.

Fonseca, E., Zelepugin, V.N., Heredia, M., 1985, Estructure of the ophiolite association of Cuba: Geothectonics, 19, 321-329.

Frost, B.R., 1985, On the stability of sulfides, oxides and native metals in serpentinite: Journal of Petrology, 26, 31-63.

García-Casco, A., Torres-Roldán, R.L., Iturralde-Vinent, M.A., Millán, G., Núñez-Cambra, K., Lázaro, C., Rodríguez-Vega, A., 2006, High pressure metamorphism of ophiolites in Cuba: Geologica Acta, 4, 63-88.

García-Casco, A., Iturralde-Vinent, M.A., Pindell, J., 2008, Latest Cretaceous collision/accretion between the Caribbean plate and Caribeana: origin of metamorphic terranes in the Greater Antilles: International Geology Review, 50, 781-809.

Garuti, G., Zaccarini, F., Economou-Eliopoulos, M., 1999, Paragenesis and composition of laurite from chromitites of Othrys (Greece): implications for Os-Ru fractionation in ophiolitic upper mantle of the Balkan peninsula: Mineralium Deposita, 34, 312-319.

Gervilla, F., Proenza, J.A., Frei, R., González-Jiménez, J.M., Garrido, C.J., Melgarejo, J.C., Meibom, A., Díaz-Martínez, R., Lavaut, W., 2005, Distribution of platinum-group elements and Os isotopes in chromite ores from Mayarí-Baracoa Ophiolilte Belt (eastern Cuba): Contributions to Mineralogy and Petrology, 150, 589-607.

González-Pontón, R., 1998, Mineralizaciones cromíticas de la asociación ofiolítica de Camagüey, Cuba (resumen), en III Congreso Cubano de Geología y Minería: La Habana, Centro Nacional de Información Geológica (CNIG), 281-283.

González-Pontón, R., 2009, Cromo Camagüey (proyecto de colaboración con el ALBA), en Tercera Convención Cubana de Ciencias de la Tierra: La Habana, Cuba, Sociedad Cubana de Geología, memorias en CD-ROM, ISBN 978-959-7117-19-3.

Iturralde-Vinent, M.A., 1989, Role of ophiolites in the geological structure of Cuba: Geotectonics, 23, 4, 332-341.

Iturralde-Vinent, M.A., 1996, Geología de las ofiolitas de Cuba, en IturraldeVinent M.A. (ed.), Ofiolitas y arcos volcánicos de Cuba: Miami, E.U.A., First Contribution IGCP Project 364, 83-120.

Iturralde-Vinent, M.A., 1998, Sinopsis de la constitución geológica de Cuba: Acta Geológica Hispánica, 33, 9-56.

Iturralde-Vinent, M.A., 2001, Field guide to the former caribbean plate boundary Camagüey, Central Cuba, in 4th Cuban Geological and Mining Congress, 2000: La Habana, Cuba, Sociedad Cubana de Geología, 1-16.

Khudoley, K., Meyerhoff, A.A., 1971, Paleogeography and geology history of the Greater Antilles: Geological Society of America Memoir, 129, 1-199.

Klein, F., Bach, W., 2009, Fe-Ni-Co-O-S phase relations in peridotite-seawater interactions: Journal of Petrology, 50, 37-59. 
Knipper, A., Cabrera, R., 1974, Tectonics and historical geology of the articulation zone between the mio- and eugeosyncline and the hyperbasite belt: Contribution to the Geology of Cuba, 15-77.

Kravchenco, G.G., Vázquez, S.O., 1985, Estructura y perspectivas cromíferas de algunas regiones de los macizos ultrabásicos de Cuba: Ciencias de la Tierra y del Espacio, 10, 37-55.

Lavandero, R.M., Estrugo, M., Santa Cruz-Pacheco, M., Bravo, F., Melnikova, A.A., Cabrera, R., Trofimov, V.A., Romero, J., Altarriba, I., Alvarez, P., Aniatov, I.I., Badamgavin, B., Barishev, A.N., Carrillo, D.J., Cazañas, X., Cuellar, N., Dovbnia, A.V., Formell, F., García, M., González, D., Gue, G.G., Janchivin, A., Krapiva, L.J., López, J., Lozanov, I., Montenegro, J., Pantaleon, G., Stefanov, N., Vázquez, O., Zaagoskin, A.M., Zhidkov, A.Ya., 1988, Carta geológico-minera Mapa de yacimientos metálicos y aguas minerales de la República de Cuba, escala 1:500,000: Ciudad de La Habana, Cuba, Ministerio de la Industria Básica, Instituto de Geología y Paleontología, 1 mapa.

Leblanc, M., Nicolas, A., 1992, Les chromitites ophiolitiques: Chronique de la Recherche Miniére, 507, 3-25.

Leblanc, M., Violette, J.F., 1983, Distribution of aluminum-rich and chromiumrich chromite pods in ophiolite peridotites: Economic Geology, 78, 293-301.

Matsumoto, I, Arai, S., Yamauchi, H., 1997, High-Al podiform chromitites in dunite-harzburgite complexes of the Sangun Zone, central Chugoku district, Southwest Japan: Journal of Asian Earth Sciences, 15, 295-302.

Murashko, V.I., Lavandero, R.M., 1989, Chromite in hyperbasite belt of Cuba: International Geology Review, 31, 90-99.

Proenza, J.A., Melgarejo, J.C., 1998, Una introducción a la metalogenia de Cuba bajo la perspectiva de la tectónica de placas: Acta Geologica Hispánica, 33, 89-132.

Proenza, J., Gervilla, F., Melgarejo, J.C., 1999a, La Moho Transition Zone en el macizo ofiolítico Moa-Baracoa (Cuba): un ejemplo de interacción magma/ peridotita: Revista de la Sociedad Geológica de España, 12, 309-327.

Proenza, J.A., Gervilla, F., Melgarejo, J.C., Bodinier, J.L., 1999b, Al- and Crrich chromitites from the Mayarí-Baracoa Ophiolite Belt (eastern Cuba): consequence of interaction between volatile-rich melts and peridotite in suprasubduction mantle: Economic Geology, 94, 547-566.

Proenza, J., Solé, J., Melgarejo, C., 1999c, Uvarovite in podiform chromitite: the Moa-Baracoa ophiolitic massif, Cuba: Canadian Mineralogist, 26, 979-990.
Proenza, J.A., Ortega-Gutiérrez, F., Camprubí, A., Tritlla, J., Elías-Herrera, M., Reyes-Salas, M., 2004a, Paleozoic serpentinite-enclosed chromitites from Tehuitzingo, (Acatlán Complex, southern Mexico): a petrological and mineralogical study: Journal of South American Earth Sciences, 16, 649-666.

Proenza, J.A., Escayola, M., Ortiz, F., Pereira, E., Correa, A.M., 2004b, Dunite and associated chromitites from Medellín (Colombia) (resumen), en: 32nd International Geological Congreso: Florencia, Italia, CD-ROM.

Rodríguez, R., Santa Cruz-Pacheco, M., Navarrete, M., Fonseca, E., de Albear, J.F., 1997, Las cromititas podiformes en las ofiolitas de Cuba, en Furrazola, G.F., Nuñez-Cambra, K.E. (eds.), Estudios sobre Geología de Cuba: Ciudad de La Habana, Cuba, Instituto de Geología y PaleontologíaCentro Nacional de la Información Geológica, 301-314.

Semionov, Y.L., 1968, Yacimientos cromiticos de Cuba, Revista Tecnológica, 3, 17-30.

Thayer, T.P., 1942, Chrome resources of Cuba: U.S. Geological Survey Bulletin 935-A, 1-74.

Thayer, T.P., 1969, Gravity differentiation and magmatic re-emplacement of podiform chromite deposits: Economic Geology Monograph, 4, 132-146.

Zaccarini, F., Proenza, J.A., Ortega-Gutiérrez, F., Garuti, G., 2005, Platinumgroup minerals in ophiolitic chromitites from Tehuitzingo (Acatlán complex, southern Mexico): implications for post-magmatic modification: Mineralogy and Petrology, 84, 147-168.

Zhou, M.F., Robinson, P.T., Bai, W.J., 1994, Formation of podiform chromitites by melt/rock interaction in the upper mantle: Mineralium Deposita, 29, 98-101.

Zhou, M.F., Yumul, G.P., Malpas, J., Sun, M., 2000, Comparative study of platinum-group elements in the Coto and Acoje blocks of the Zambales Ophiolite Complex, Philippines: Island Arc, 9, 556-564.

Zhou, M.F., Robinson, P.T., Malpas, J., Aitchison, J., Sun, M., Bai, W.J., Hu, X.F., Yang, J.S., 2001, Melt/mantle interaction and melt evolution in the Sartohay high-Al chromite deposits of the Dalabute ophiolite (NW China): Journal of Asian Earth Sciences, 19, 517-534.

Manuscrito recibido: 19/11/2009

Manuscrito corregido recibido: 22/12/2009

Manuscrito aceptado: 28/12/2009 